\title{
Le vocabulaire des directions cardinales dans les dialectes du continuum cri-innu-naskapi- atikamekw
}

The vocabulary of cardinal directions in the Cree-Innu-Naskapi-Atikamekw dialectal continuum

El vocabulario de las direcciones cardinales en los dialectos del continuum criinnu-naskapi-atikamekw

\section{Vincent Collette}

\section{OpenEdition \\ Journals}

Édition électronique

URL : https://journals.openedition.org/jsa/14009

DOI : 10.4000/jsa. 14009

ISSN : 1957-7842

\section{Éditeur}

Société des américanistes

Édition imprimée

Date de publication : 31 décembre 2014

Pagination : 7-44

ISSN : 0037-9174

Référence électronique

Vincent Collette, «Le vocabulaire des directions cardinales dans les dialectes du continuum cri-innunaskapi-atikamekw », Journal de la Société des américanistes [En ligne], 100-2 | 2014, mis en ligne le 01 janvier 2016, consulté le 03 septembre 2022. URL : http://journals.openedition.org/jsa/14009 ; DOI https://doi.org/10.4000/jsa. 14009 


\title{
LE VOCABULAIRE DES DIRECTIONS CARDINALES DANS LES DIALECTES DU CONTINUUM CRI-INNU-NASKAPI-ATIKAMEKW
}

\author{
Vincent COLLETTE*
}

L'objectif de cet article est de présenter une étymologie comparée du vocabulaire des directions cardinales dans les langues et dialectes appartenant à la branche géographique de l'algonquien du Centre et, plus particulièrement, dans les dialectes appartenant au continuum cri-innu-naskapi-atikamekw. L'analyse de la morphologie et de la sémantique des mots (particules, verbes et substantifs) qui servent à exprimer les directions cardinales montre que ces langues font usage de quatre sources de lexicalisation pour nommer les directions cardinales (cycle du soleil, direction du vent, cours des rivières et déité éolienne/cardinale). Les données des dialectes plus orientaux du continuum cri-innu-naskapi-atikamekw indiquent que, dans les sociétés traditionnelles, le référent qui sert de source de lexicalisation des termes de directions (cours des rivières et cycle du soleil) est souvent plus saillant que la direction elle-même, et que certains changements économiques, sociaux, culturels ou religieux, provoqués par la présence des personnes et des institutions de la société industrielle dominante, peuvent accélérer le chevauchement, puis le remplacement des termes dans le champ lexical des directions cardinales. [Mots-clés : langues algonquiennes, continuum cri-innu-naskapiatikamekw, directions cardinales, sources de lexicalisation, morphologie, mythologie.]

The vocabulary of cardinal directions in the Cree-Innu-Naskapi-Atikamekw dialectal continuum. The aim of this article is to describe the vocabulary of cardinal directions in languages belonging to the geographical branch of Central Algonquian and, particularly, for the dialects belonging to the Cree-Innu-Naskapi-Atikamekw continuum. The analysis of the morphology and the semantics of the words (particles, verbs and nouns) which express cardinal directions shows that these languages use four sources of lexicalization in order to name the cardinal directions: sun cycle, wind direction, river flow, wind/cardinal deity. The data from the Eastern dialects of the Cree-Innu-Naskapi-Atikamekw continuum indicate that in traditional societies the referent which serves as the source of lexicalization for cardinal directions (that is, river flow and sun cycle) is more salient than the direction itself and that some economic,

* First Nations University of Canada, Department of Indigenous Languages, Arts and Culture (DILAC), 1 First Nations Way, Regina, Saskatchewan, Canada, S4S 7K2 [vcollette@hotmail.com].

Journal de la Société des américanistes, 2014, 100-2, pp. 7-44. C Société des américanistes. 
social, cultural and religious changes, provoked by the presence of persons and institutions belonging to the dominant society may accelerate lexical overlaps and replacement in the lexical domain of cardinal directions. [Key words: Algonquian languages, cree-innu-naskapi-atikamekw continuum, cardinal directions, sources of lexicalisation, morphology, mythology.]

El vocabulario de las direcciones cardinales en los dialectos del continuum cri-innu-naskapi-atikamekw. El objetivo de este artículo es presentar una etimología comparada del vocabulario de los puntos cardinales en las lenguas que pertenecen al territorio geográfico algonquino del Centro, particularmente en los dialectos del continuum cri-innu-naskapi-atikamekw. El análisis de la morfología y de la semántica de las palabras (las partículas, los verbos y los sustantivos) que sirven para expresar los puntos cardinales, muestra que estas lenguas hacen uso de cuatro fuentes de lexicalización para nombrar los puntos cardinales: el ciclo del sol, la dirección del viento, el curso de los ríos y la deidad eólica/cardinal. Los datos tomados de los dialectos más orientales del continuum cri-innu-naskapi-atikamekw indican que, en las sociedades tradicionales, el referente que sirve de fuente de lexicalización de los términos relacionados con los puntos cardinales (como el curso de los ríos y el ciclo del sol), frecuentemente es más sobresaliente que la dirección misma y que algunos cambios económicos, sociales, culturales o religiosos - provocados por la presencia de personas e instituciones de la sociedad industrial dominante pueden acelerar las transposiciones y, más tarde, el reemplazo de los términos que hacen parte del campo lexical de los puntos cardinales. [Palabras claves: lenguas algonquinas, continuum cri-innu-naskapi-atikamekw, direcciones cardinales, fuentes de lexicalización, morfología, mitología.]

C'est un lieu commun, du moins pour les locuteurs de langues indoeuropéennes, de parler de « quatre » points cardinaux et de deux axes : NORD-SUD et EST-OUEST [Les petites capitales indiquent les directions et les places cardinales comme référent non linguistique]. Or cet arrangement n'est en rien universel, car dans certaines langues comme le zoulou ou le sésotho (langues bantoues), pour ne nommer que celles-là, on trouve seulement trois « points » cardinaux (Brauner 1998). De plus, des auteurs comme Brown (1983) ou Heine (1997) ont démontré que certaines langues avaient établi ou même renouvelé leur système en fonction des particularités géographiques, écologiques ou cosmologiques locales. Ailleurs encore, une langue peut emprunter ses termes de directions cardinales à une langue voisine plus prestigieuse. En effet, les termes de direction ne relèvent pas du lexique de base et sont donc plus facilement empruntables. À titre d'exemple, on peut noter que les noms des points cardinaux en français furent empruntés à des marins parlant le moyen anglais qui fréquentaient la côte de Normandie pendant la deuxième moitié du XII ${ }^{\mathrm{e}}$ siècle (Gutmans 1970, pp. 150 sq.). Ces mots passèrent à l'espagnol et au portugais par l'intermédiaire du français.

Cet article, qui a pour objectif de documenter le lexique des directions cardinales dans les dialectes du continuum cri-innu-naskapi-atikamekw, 
s'organise comme suit. Dans la section 1, nous définissons les concepts de « point », de « direction » puis d' " orientation cardinale » et montrons que la notion de quadricardinalité est ancienne et solidement ancrée dans la mythologie et les enseignements traditionnels des peuples algonquiens, de même que dans la façon dont ces peuples répartissent l'espace domestique. Nous exposons, dans la section 2, certains éléments de morphologie des langues algonquiennes. Après avoir présenté nos objectifs de recherche, nous présentons et discutons, dans la section 3, les sources de lexicalisation (cycle du soleil, direction des vents, cours des rivières, déité éolienne/cardinale) des mots servant à exprimer les directions/orientations cardinales dans une douzaine de dialectes et langues appartenant à la branche géographique de l'algonquien du Centre. Enfin, nous analysons, dans la section 4, certains microsystèmes cardinaux plus en profondeur, ce qui nous permet de mettre en lumière la nature et la direction des changements sémantiques en cause pour le champ lexical des termes de directions cardinales (Figure 1).

\section{QuADricardinalité, POINTS, PlaCES ET DiRECTIONS CARDINALES}

Dans cet article, nous considérons un point cardinal comme un terme de géographie qui renvoie à un système de localisation fondé sur un point fixe, le Nord magnétique. Toutefois, puisque la notion de « point cardinal » est directement liée à la découverte du magnétisme et au développement des techniques de navigation, nous avançons qu'il faut faire une distinction entre les notions de " point cardinal» et de "direction cardinale », cette dernière reposant sur l'observation de phénomènes naturels comme le cycle solaire, la présence d'une étoile dans le ciel ou la provenance des vents. Ces points de repère ne sont pas tout à fait absolus, puisqu'ils ne permettent pas de repérage immédiat en territoire inconnu, par temps couvert ou sans vent, ou encore pendant une certaine période de l'année. Pour éviter de plaquer une vision occidentale de la cardinalité sur les données du domaine algonquien, nous proposons de penser les directions cardinales non pas comme des points cardinaux fixes, mais, plutôt, comme des directions physiquement inatteignables dans le tracé solaire : l'est est la direction où le soleil se lève ; l'ouest est celle où il se couche; alors que le sud est la direction où vont les morts et d'où viennent les oiseaux migrateurs au printemps (Hallowell 1955, p. 190). Ces directions cardinales ne correspondent pas parfaitement aux points cardinaux d'une boussole puisqu'elles sont fondées sur l'observation de phénomènes atmosphériques ou physiques qui peuvent varier selon les saisons, les particularités géographiques locales ou encore l'axe d'inclinaison de la terre à une certaine latitude. Enfin, nous réservons le concept de «place cardinale » aux termes qui ont un point de référence relatif et atteignable comme le cours d'une rivière ou la cime d'une montagne. Nous y reviendrons dans la sous-section 4.3. 


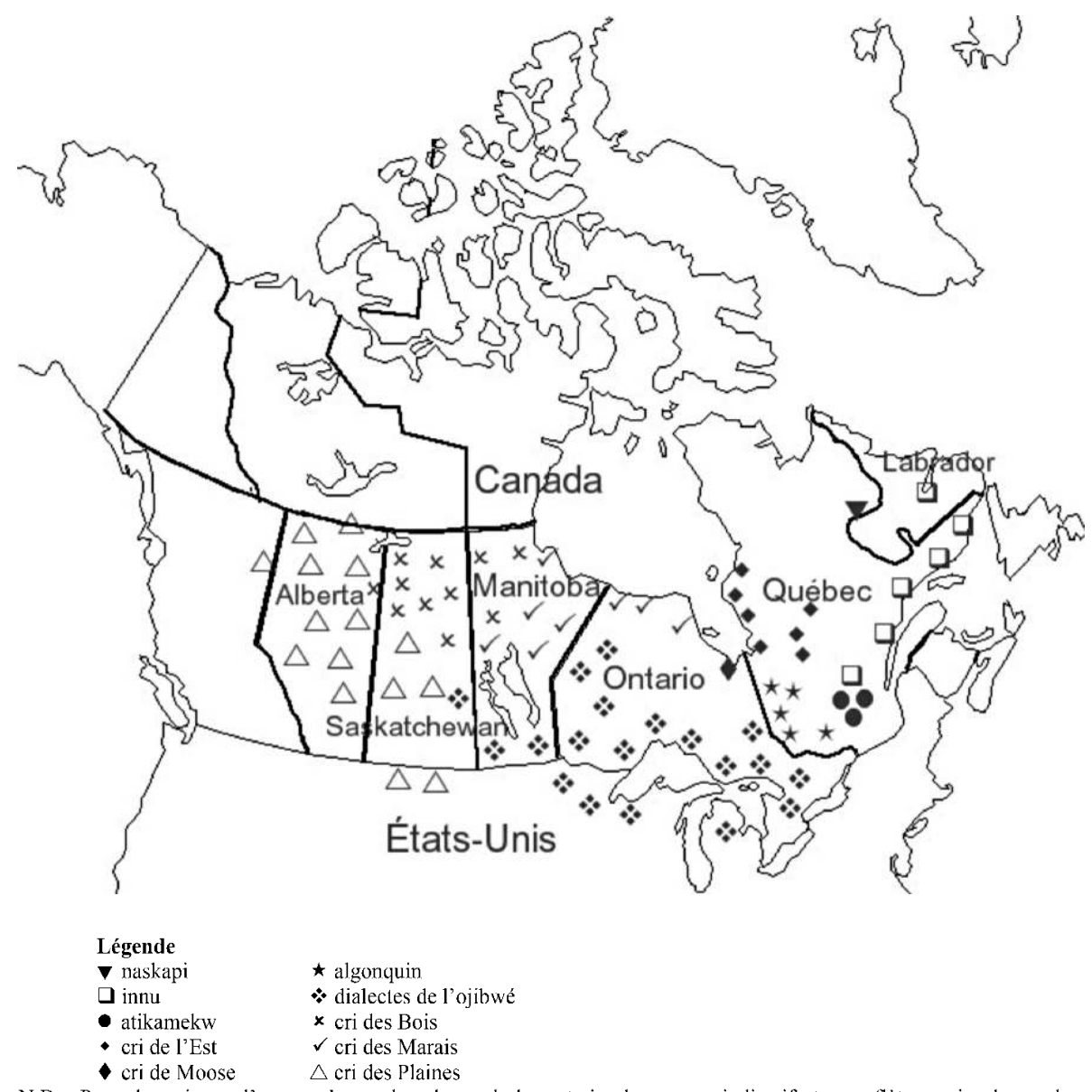

N.B. : Pour des raisons d'espace, le nombre de symboles est simplement un indicatif et ne reflète en rien le nombre exact des communautés linguistiques présentes sur le territoire.

FIG. 1 - Répartition géographique des dialectes des continuums cri-innu-naskapi-atikamekw et ojibwé-algonquin.

Puisque l'on a affaire à des cultures orales sans système ancien d'écriture, l'examen de certains mythes et des relations structurales sur lesquelles ils reposent s'avère important pour la compréhension des données linguistiques. Il existe plusieurs versions de la création des quatre vents (ou wind-brothers) dans la littérature sur les langues algonquiennes ${ }^{1}$. Le fait le plus marquant est que les vents sont considérés comme des déités qui vivent aux quatre coins de la terre et qui président aux saisons, au climat, de même qu'à la vie spirituelle et physique humaine. Nous définissons ce concept de "déité » non comme une notion 
religieuse relevant d'un stade inférieur mais comme une entité abstraite au statut divin. Ces déités éoliennes sont bien connues dans la littérature sur la mythologie algonquienne (voir Brinton 1868; Jones 1911, 1939 ; Radin et Reagan 1928 ; Johnston 1976; Brown 1977 ; Tanner 1979). On retrouve, dans l'imposante mythologie ojibwé, des références explicites au sujet de la création des déités cardinales et de leurs noms propres, comme l'indique le passage suivant : "Kitche Manitou created four incorporeal beings: Zhawano (South), Keewatin (North), Waubun (Morning or East), and Ningobianong (Evening or West). He assigned each one to a quarter of the world and to a portion of time. To each he gave a power over life to be exercised with wisdom » (Johnston 1976, pp. 27-28). Certains noms de déités cardinales correspondent à des verbes impersonnels ou à des substantifs dans les langues algonquiennes. En ojibwé par exemple, le nom Waubun correspond au verbe impersonnel waaban «c'est l'aube » (II) ${ }^{2}$, alors que Keewatin correspond à giiwetin « il vente du nord » (II) en ojibwé, mais à tshiuetin « il vente du nord » (II), « le Nord » (NI) en innu. Comme l'indique ce dernier exemple, il ne semble pas y avoir, dans la cosmologie algonquienne, de différence nette entre le vent venant d'une direction cardinale donnée et la direction elle-même, ni même entre la déité éolienne et la direction cardinale où elle réside, et c'est pourquoi nous utiliserons ici le terme indistinct de « déité éolienne/cardinale ».

Les mythes de création des vents, et les oppositions structurales et symboliques sur lesquelles ils reposent, indiquent que la notion de déités éoliennes/cardinales résidant dans les quatre portions de l'horizon - et s'intégrant dans un réseau d'oppositions cosmologiques qui articule la répartition des saisons, la généalogie, de même que certains traits psychologiques ou moraux est d'une grande antiquité dans le domaine algonquien. Dans la section 3, nous analyserons plus en profondeur certains des termes de direction qui semblent dérivés d'un nom de déité. Dans la section suivante, nous présentons les sources utilisées ainsi que certains éléments de morphologie linguistique.

\section{2. ÉLÉMENTS DE MORPHOLOGIE DES LANGUES ALGONQUIENNES}

Toutes les langues et dialectes ${ }^{3}$ algonquiens partagent un ancêtre commun, le proto-algonquien (PA ci-après), dont la phonologie fut partiellement reconstruite par Bloomfield (1946) au moyen de la méthode comparative en linguistique historique. Les dialectes qui constituent notre échantillon font partie de la branche géographique de l'algonquien du Centre. Cette dernière ne forme pas un groupe génétique distinct puisque, en regard du PA, il n'y a pas plus de distance génétique entre une langue de l'algonquien du Centre (par exemple l'innu) et une de l'algonquien de l'Ouest (par exemple le blackfoot; Valentine 2001, p. 14). Les langues qui forment l'algonquien de l'Est diffèrent, elles, génétiquement de celles appartenant à l'algonquien du Centre ou de l'Ouest, et elles sont donc plus 
distantes linguistiquement. Les dialectes et sous-dialectes que nous étudierons dans cet article proviennent essentiellement de ce qui est appelé en linguistique algonquienne le continuum dialectal cri-innu-naskapi-atikamekw (CINA ciaprès). Il s'agit d'un ensemble relativement homogène de dialectes qui s'étend de l'Alberta jusqu'au Labrador. Il importe de noter que l'aire algonquienne du Centre n'est pas tout à fait homogène sur le plan écologique car les différentes communautés linguistiques à l'étude habitent la forêt boréale, certaines régions côtières, les plaines canadiennes, de même que la toundra forestière. Comme nous allons le voir dans les sous-sections 3.2 et 3.3, environnement et nomenclature des directions cardinales sont intimement liés comme l'indique, par exemple, la présence, dans presque toutes les langues de l'algonquien du Centre septentrional, de termes ayant le vent du nord comme source de lexicalisation pour la direction NORD, alors que dans les régions plus méridionales de l'aire algonquienne les sources de lexicalisation diffèrent pour la direction NORD.

Enfin, même si cet article porte essentiellement sur les dialectes du continuum CINA, nous avons aussi intégré les données provenant du continuum anishnabe, lequel regroupe l'algonquin (du Québec et de l'Ontario) et l'ojibwé (Manitoba, Ontario et États-Unis), afin de donner une certaine profondeur comparative à notre description. Les langues des deux continuums susmentionnés forment la branche géographique de l'algonquien du Centre avec le mesquakie, le menominee et le kickapoo. Les données provenant du PA (Goddard 1983; Hewson 1993 ; Proulx 1986) ont été utilisées pour mieux comprendre comment s'est effectué le changement linguistique. Toutes les données présentées ici le sont dans l'orthographe de la source originale. [Les phonèmes vocaliques longs et tendus sont orthographiés soit avec un accent circonflexe $(\hat{a})$, soit par redoublement $(a a)$.] Le lecteur pourra consulter l'Annexe 1 qui contient les sources bibliographiques consultées et les codes utilisés dans cet article.

Avant de procéder à l'analyse des données, il importe d'avoir une idée de la structure interne du mot dans les langues algonquiennes. Les langues algonquiennes sont polysynthétiques (Sapir 1970 [1921] ; Bloomfield 1970 [1933]; voir Evans et Sasse 2002) et non configurationnelles (Hale 1989). Les verbes sont fléchis pour la personne, le nombre, l'obviation ${ }^{4}$ (personnes rapprochée et obviée), le temps et le mode. Les substantifs le sont pour la personne (possesseur/possessum), le nombre et l'obviation. Les particules sont invariables. Le radical verbal comporte trois positions: initiale, médiane et finale (voir Goddard 1990). Les éléments figurant dans la position initiale permettent d'exprimer des procès, des états et des qualités, alors que ceux qui apparaissent en position médiane indiquent des entités et la forme bi- ou tri-dimensionnelle d'un objet (Drapeau et Lambert-Brétière 2011). Les finales se scindent en deux classes. Les finales concrètes indiquent un procès ou le moyen utilisé dans un procès, alors que les finales abstraites indiquent l'appartenance du verbe à l'une des quatre classes verbales (TA, TI, IA et II). Les verbes se classent selon les paramètres de la 
«transitivité » et du genre du sujet (verbes intransitifs) et de l'objet (verbes transitifs), soit : TA verbe transitif avec objet animé ; TI verbe transitif avec objet inanimé ; IA verbe intransitif avec sujet animé ; et II verbe intransitif avec sujet inanimé. Le statut de la finale abstraite est controversé et nous la garderons attachée dans la présente étude, mis à part pour les exemples ci-dessous. Seule la médiane est optionnelle. Un radical consiste en un mot moins sa flexion. S'il n'y a que deux composantes dans un radical, ce sera l'initiale et la finale. C'est la structure des particules adverbiales de lieux, des verbes inanimés intransitifs (II) et des substantifs (NA, NI) qui nous importera dans cet article. Voici des exemples en cri de l'Est (dialecte du Nord) qui permettront d'expliciter ce qui vient d'être exposé. Dans les exemples suivants, le mode et le temps sont neutres par défaut et donc non marqués morphologiquement.

Exemple 1. a. wâpin : c'est l'aube (II)

\begin{tabular}{|c|c|c|}
\hline $\begin{array}{l}\text { /wâpin }{ }^{\mathrm{w}}- \\
\text { initiale } \\
\text { être.aube }\end{array}$ & $\begin{array}{l}\varnothing- \\
\text { finale } \\
\text { II }\end{array}$ & $\begin{array}{l}\varnothing / \\
\text { flexion } \\
3^{\mathrm{e}} \text { inanimé sg. }\end{array}$ \\
\hline \multicolumn{3}{|c|}{$\begin{array}{l}\text { b. wâpinîwâw : il vente de l'est (II) (« il vente de l'aube, du solei } \\
\text { levant ») }\end{array}$} \\
\hline $\begin{array}{l}\text { /wâpin }{ }^{\mathrm{w}} \text { - } \\
\text { initiale } \\
\text { être.aube }\end{array}$ & $\begin{array}{l}\hat{\imath} w \hat{a}- \\
\text { finale } \\
\text { venter. II }\end{array}$ & $\begin{array}{l}w / \\
\text { flexion } \\
3^{\mathrm{e}} \text { inanimé sg. }\end{array}$ \\
\hline
\end{tabular}

La plupart des directions ou places cardinales sont exprimées au moyen d'une particule (PT) ${ }^{5}$ adverbiale de lieu indécomposable morphologiquement (exemple 2), d'une particule adverbiale de lieu dérivée d'un radical verbal (exemple 3b), ou encore d'un substantif dérivé (exemple 4b). Dans les deux derniers cas, la source de lexicalisation est toujours indiquée par le radical verbal qui se trouve en position initiale. Voici quelques exemples en cri de l'Est (dialecte du Nord) :

Exemple 2. achishtuw : du côté ouest (PT adverbiale de lieu) /achituw/

Exemple 3. a. wâpin ${ }^{\mathrm{w}}$ : c'est l'aube (II)

b. wâpinûtâhch: du côté est (« du côté de l'aube ») (PT adverbiale de lieu)

Iwâpin ${ }^{w}$ -

initiale

être.aube ^tâhchl

locatif

du.côté.de IA 
Exemple 4. a. chîwâtin : il vente de l'ouest (II)

b. chîwâtinishish : le nord-ouest (NI)

lchîwâtin- ishish- Ø/

ouest- diminutif- $\quad 3^{\mathrm{e}}$ inanimé sg.

Comme l'indique l'exemple $3 \mathrm{~b}$, on trouve, dans plusieurs particules adverbiales de lieu, un locatif (^hch ou -^tâhch - âhtâhch, -etâhch) qui termine le mot. L'étymologie et l'analyse diachronique de ces locatifs ont été débattues dans les années 1980 (voir Proulx 1980, 1984 ; Goddard 1983). Selon nous, le suffixe allongeant -^tâhch - qui présente des allomorphes avec ou sans /h/ initial et une voyelle de connexion /a:/ ou /e/ selon les langues ou dialectes - est un suffixe locatif qui revêt le sens de " du côté de ». Ainsi, en cri de l'Est (dialecte du Sud) pîsimw-etâhc signifie «au/dans le sud», littéralement « du côté du soleil». Les réflexes du locatif $\mathrm{PA} *-n k$ sont réguliers $(-n g,-h k,-k k,-h c h$, chacun apparaissant avec une voyelle brève de connexion) pour l'ensemble des dialectes à l'étude. À présent que ces principes de formation de mots ont été exposés, penchons-nous sur la description et l'analyse des sources de lexicalisation des termes exprimant les directions et les places cardinales.

\section{LES SOURCES DE LEXICALISATION DES DIRECTIONS ET PLACES CARDINALES}

Dans ce qui suit, nous allons décrire les sources de lexicalisation présentes dans notre échantillon, c'est-à-dire le cycle du soleil (3.1), la direction des vents (3.2), le cours des rivières (3.3) et certains noms de déités ou leur lieu de résidence (3.4). Afin d'alléger le texte, l'ensemble des données linguistiques est présenté dans l'Annexe 2. Notre hypothèse de travail est la suivante : étant donné que les peuples algonquiens septentrionaux pratiquaient la chasse, la pêche et la cueillette et misaient crucialement, pour leurs déplacements saisonniers et les succès à la chasse, sur un savoir écologique détaillé et des prescriptions rituelles strictes envers les déités éoliennes/cardinales, on a des raisons de penser que les référents des sources de lexicalisation des mots exprimant les directions ou les places cardinales sont naturellement et/ou culturellement plus saillants que les directions elles-mêmes puisque les termes de directions sont constamment renouvelés par des sources de lexicalisation différentes, ce qui expliquerait, selon Proulx (1986, p. 22), l'important degré de chevauchement et de remplacement lexical dans cette portion du vocabulaire. Si cette hypothèse s'avérait juste on pourrait s'attendre à ce qu'il y ait une certaine corrélation entre transformations socioéconomique, linguistique et culturelle et changements dans la structure et dans les sources de lexicalisation du vocabulaire des directions cardinales. Cette hypothèse de travail servira de trame de fond à notre analyse. 


\subsection{Cycle du soleil}

Le cycle du soleil constitue une importante source de lexicalisation pour indiquer l'EST dans les langues du monde. En proto-indo-européen, par exemple, la racine *es-, qui désignait à l'origine un être animé et vénéré dans la spiritualité des premiers habitants de l'Eurasie, a donné le latin aurora (via *ausosa) duquel sont issus le substantif « aurore » et l'adjectif « austral » en français ; l' « ouest » est issu du latin vesper (< grec hésperos « soir ») (Gutmans 1970, pp. 150 sq.). Il s'agit d'un cas de synesthésie, ou extension sémantique allant du concret (le soir) à l'abstrait (l'ouest) (Hock 1991, pp. 289-290).

Nous avons relevé trois stratégies de formation de mots qui ont le cycle solaire comme source de lexicalisation. Le lecteur pourra consulter l'Annexe 2 afin de se donner une idée de l'uniformité qui caractérise les dialectes de l'algonquien du Centre quant à cette source de lexicalisation.

- Premièrement, on notera l'utilisation d'un réflexe de PA *wa.panwi « c'est l'aube » auquel on ajoute un des locatifs issus de PA *-nk ou de *-weta.hke. ${ }^{6}$. Ces réflexes présentent la répartition géographique la plus importante dans les langues de notre échantillon : waapinuuhch (NI) (naskapi); wâpanûtâhch (PT) (cri de l'Est, dialecte du sud-intérieur); wa:panokk (PT) (atikamekw); waabanong (PT) (ojibwé, algonquin) et wâpanohk (PT) (cri des Plaines) ;

- Deuxièmement, notre échantillon contient aussi des cas d'utilisations du substantif animé pîsimw «astre, soleil » (ou de la médiane correspondante) duquel on dérive des particules adverbiales de lieu indiquant l'opposition SUD/NORD ou parfois les termes pour SUD et OUEST. Sur le plan morphologique, l'opposition est obtenue par ajout d'une initiale âtim- " à l'opposé » à la médiane -âpîsimw-, soit : pîsimw-âhtâhch «dans, au sud » (PT)/âtim-âpîsimw « dans, au nord» (PT) (litt. «à l'opposé du soleil») (cri de l'Est, dialecte du Nord) ; aatimaapiisimuuhch « l'ouest » (NI) (naskapi);

Alors que cette deuxième stratégie se présente seulement en cri de l'Est et en naskapi, il existe d'autres stratégies qui sont confinées aux Cris des Plaines et des Bois, à l'ojibwé et à l'algonquin :

- Enfin, l'utilisation de particules adverbiales de lieu dont l'une a la position du soleil à midi (zénith) comme source de lexicalisation, alors que l'autre a une condition atmosphérique découlant de la position du soleil à midi. En cri des Plaines, on trouve âpihtâkîsikâhk «dans, au sud» (PT) (de âpihtâ- "moitié » + -kîsikâ- " être le jour» + -hk « locatif ») et de kîsapwênohk « au sud, le sud» (PT) qui est obtenue à partir du radical kîsapwên ${ }^{w}$ - " faire chaud », auquel on ajoute le locatif -hk. Ces termes transparents coexistent avec pahkisimohk pahkisimohtâhk (PT) (cri des Plaines) ou epangishimodj (algonquin du Québec), une particule adverbiale de lieu dérivée d'une locution verbale dont le sens littéral est « là où il (soleil, NA) se couche » et qui signifie « à/dans l'ouest » (PT). Notons 
que le cri des Bois est le seul dialecte de notre corpus à indiquer l'est au moyen du préverbe lexical mâchi- "débuter, commencer»: mâchi-kîsikan-ohk «à/vers l'est » (litt. « là où le jour débute »). Ces verbes et locutions verbales coexistent avec d'autres particules et noms inanimés sémantiquement obscurs que nous analyserons dans la section 3.4.

\subsection{Direction du vent}

Certaines conditions atmosphériques, et tout spécialement la direction des vents, constituent une autre source importante de lexicalisation pour exprimer les directions cardinales NORD, OUEST et SUD dans les langues de notre échantillon. Nous avons relevé trois stratégies de formation lexicale.

- Tout comme la direction EST, les dialectes de notre échantillon utilisent un réflexe de $\mathrm{PA} * k i \cdot w e \cdot t e n w i$ « il vente du nord » auquel est ajouté un locatif issu de $\mathrm{PA} *-n k$ ou de *-weta.hke. : chiiwaatinuuhch (naskapi); chîwâtinûtâhch (cri de l'Est, dialecte du Nord); kìwedinong (algonquin); kîwêtinohk (cri des Plaines). Toutefois, dans certaines variantes du cri de l'Est (dialecte du Sud) chîwetinihch signifie « dans, à l'ouest » alors qu'on obtient " au, dans le nord » en ajoutant l'initiale pâshtâ- « au-delà » à chîwetin qui sert de point d'ancrage lexical oppositionnel, soit pâshtâchîwetin «au, dans le nord» qui signifie littéralement « au-delà du vent d'ouest ». Nous y reviendrons plus loin.

- L'innu, comme nous allons le voir en section 4.3, a subi une refonte presque complète de son système de directions cardinales. Les réflexes archaïques furent remplacés, pour la plupart, par des particules adverbiales ou, dans le cas de la direction SUD, par un verbe akualutin « il vente du sud (II)/Sud (NI)» présentant une extension pragmatique qui traduit une particularité éolienne propre à cette région hydrographique, soit akua- «l'autre rive » + -lutin « il vente ».

Selon Proulx (1986, p. 19), le verbe PA *ki we tenwi « il vente du nord » serait formé d'une finale déverbale *-Vtenwi « venter » liée à une initiale obscure *ki·wau moyen de la voyelle connective */e $\cdot$. Selon nous, cette analyse est contestable et nous proposons plutôt d'y voir l'initiale PA *ki we $(t)$ - " retourner chez soi » suivie de la finale indiquant l'action du vent. Ce radical simple entre dans la formation d'un nombre considérable de verbes dans les langues modernes, par exemple, chîwâw « il retourne chez lui » (IA); chîwâhyâw "il (oiseau) vole en direction sud » (IA), littéralement « il s'en retourne chez-lui en volant » (cri de

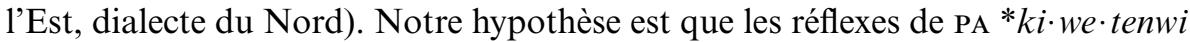
réfèrent tout simplement à l'observation multicentenaire de la trajectoire saisonnière des oiseaux migrateurs sur l'axe NORD-SUD. Cette analyse étymologique « le vent du retour » est celle offerte par un de mes jeunes informateurs de Whapmagoostui qui considérait le vent du nord comme acteur essentiel à la chasse à l'oie en automne. Ce lien entre la migration de certaines espèces aviaires et leur demeure méridionale est explicite dans un mythe ojibwé mettant en scène vents et 
oiseaux migrateurs. Voici un extrait de ce mythe présenté par Jones (1916, p. 12) : "The first-born sons of the bird-folk played ball with the North Wind and were beaten. The North Wind made goal at the west, and for that reason the wind from the east brings bad weather; the next goal was at the south, and on that account everything flees southward when the wind blows from the north. Only they that played on the side of the North Wind do not go away in winter. "Évidemment, d'un point de vue strictement linguistique, on pourrait avancer qu'il s'agit tout simplement d'un cas de réinterprétation d'une initiale obscure $* k i \cdot w$-motivé par une initiale quasi homophonique et sémantiquement transparente $* k i \cdot w e \cdot(t)-$. Nous allons voir, dans le cas des termes pour la direction SUD, que la réinterprétation est un vecteur de remplacement linguistique dans ce champ lexical.

Penchons-nous maintenant sur le cas des termes opaques qui indiquent l'OUEST en ojibwé (ningaabii-'an « l'Ouest » [NI]), en algonquin (shangàbìyan-ong "l'Ouest» [PT]) et en cri de l'Est (nikâpehan-îtâhch "l'Ouest»[PT]). Leur traitement est complexe et les données tirées de la mythologie sont peu éclairantes. Nous avons dit, dans la section 1, que Ningobianong est le nom propre de la déité cardinale de l'ouest (ou même d'un homme de médecine) existant aux côtés de Zhawano (SUD), Waubun (EST) et Keewatin (NORD) dans la mythologie ojibwé. Le premier problème qui se présente est que l'orthographe utilisée par Johnston (1976) est incorrecte. En effet, si le nom Ningobianong a bel et bien, selon lui (ibid., p. 30), le sens littéral de «l'étoile qui coule ou qui fond dans l'eau " ${ }^{7}$ alors, le deuxième $o$ devrait être /a/ -anang « étoile » et le premier $o$ devrait être /â/ comme l'indiquent les sources lexicographiques consultées. Tout au plus peut-on dire que Johnston semble avoir écrit certains phonèmes comme /a/ et /â/ avec la lettre $o$.

Pour ce qui est de l'ojibwé ningaabii'an "l'Ouest» (NI), de l'algonquin shangàbìyanong " l'Ouest » (PT) et du cri de l'Est nikâpehan-ûtâhch « l'Ouest » (PT), l'analyse comparative est entravée par des incohérences dans les correspondances vocaliques. Les problèmes rencontrés se résument comme suit :

- Bien qu'on puisse postuler que l'initiale de ces mots sont des réflexes de PA * $\theta e n k$ - «fondre » (> tihk- «fondre » en cri de l'Est), on ne peut expliquer la présence de la première voyelle longue /â/.

- Si la nasale ojibwé /ng/ de ningaabii'an « l'Ouest » (NI) était présente en PA, alors on s'attendrait à retrouver une correspondance /hk/ dans la particule nakâpehanû-tâhch « à/dans l'ouest », ce qui n'est évidemment pas le cas.

- En ce qui a trait aux réflexes - $(\hat{a}) b \hat{\imath}-,-(\hat{a}) p e$ - de la médiane PA *-epy $(e:)(k)$ « liquide, eau », il est vrai qu'il existe une correspondance entre l'ojibwé /i/ et le cri de l'Est /e/, mais la présence de la voyelle longue initiale /â/ de ce morphème reste obscure.

- Il existe une correspondance entre l'aspirée / $\mathrm{h} / \mathrm{du}$ cri de l'Est et la glottale de l'ojibwé du sud-ouest I'/, ce qui explique la différence entre -âpehan (cri de l'Est) et -âpîan (ojibwé) ; toutefois pour ce qui est de la signification « venter », seul le cri de l'Est présente une finale II en - $(a)$ han, laquelle apparaît dans une poignée de 
verbes impersonnels. Du reste, l'inexistence d'une finale de ce type en ojibwé pourrait expliquer pourquoi ningaabii'an « l'Ouest » (NI) exprime seulement une direction et non un vent en synchronie. En somme, les réflexes modernes semblent contenir une médiane pour « eau » et une finale pour « venter » mais cela reste incertain puisque la signification abstraite (et donc la source de lexicalisation) de ces mots est désormais opaque.

Dans un autre ordre d'idées, on retrouve dans la mythologie le nom Kapeyun « vent de l'ouest » qui fut donné à Madjiigawia à la suite d'un épisode héroïque dans le monde des ours situé à l'Occident. Le mot Kapeyun, qui présente les variantes Kabun, Kabeyan et Kabeyang (Coulter et Turner 2000), semble relié, sur les plans morphologiques et sémantiques, aux termes pour OUEST en ojibwé et en cri de l'Est. Il est possible que Kabeyun et ses variantes soient des corruptions du verbe « il vente de l'ouest », car les noms de héros mythiques ou de personnages légendaires ont tendance à subir d'importants changements phonologiques; le nom du démiurge Nenabozho, par exemple, présente la variante Wenabozho (et d'autres avec une nasale $/ \mathrm{m} /$ initiale) dans les différents dialectes ojibwés (Nichols et Nyholm 1995).

En résumé, il faudra attendre que d'autres recherches en dialectologie soient menées pour que l'analyse morphologique et étymologique de ningaabii'an/nakâpehan sorte de l'obscurité dans laquelle elle se trouve présentement. Tout ce que l'on peut affirmer avec certitude, c'est qu'en synchronie seul nakâpehanû-tâhch « à/dans l'ouest » a un radical verbal exprimant le vent d'ouest comme source de lexicalisation. Dans l'ojibwé ningaabii'an « l'Ouest» (NI), le suffixe -'an correspondant au cri -ahan « venter » n'est vraisemblablement plus productif. Dans les autres dialectes et langues, on retrouve ce radical opaque sémantiquement, suivi d'un réflexe du locatif $\mathrm{PA} *$-nk ou de *-weta.hke. soit : nakâpehano-kk «côté ouest»(PT) (atikamekw); nikâpâhân̂ิ-tâhch «c'est à l'ouest » (PT) (cri de l'Est, dialecte du Nord, Chisasibi); nakapehâno-hk « dans l'ouest » (PT) (cri de Moose); nakapêhano-hk «dans l'ouest » (PT) (cri des Plaines); shangàbìyano-ng « l'Ouest » (PT) (algonquin).

\subsection{Cours des rivières}

Quelques dialectes de notre échantillon ont le cours des rivières comme source de lexicalisation pour les directions OUEST et EST. Dans les écrits du XVII ${ }^{\mathrm{e}}$ siècle, ces particules n'indiquent pas une direction cardinale. Bonaventure Fabvre (1970 [1695], pp. 135 et 184) note mamits, mamik « A ual en desc(en)dant La Riu(iere) » ainsi que natimich « A mont, en haut La Riui(er)e » et natimitac « un peu uers Le haut du fleuue ». Certains dialectes, comme l'innu, ont lexicalisé ces particules adverbiales de lieu puisque l'aval réfère (dans le Sud-Ouest de l'aire innue) à la direction EST, et l'amont à la direction OUEST. C'est ainsi que l'on retrouve, dans tous les sous-dialectes de l'innu, les particules adverbiales mâmîht mamit « aval, 
à l'est » et natimit « amont, à l'ouest », puisque plusieurs communautés innues sont situées à l'aval des grandes rivières se jetant dans le fleuve Saint-Laurent. Toutefois, les sources consultées indiquent que cette extension pragmatique de la signification littérale de la particule adverbiale de lieu est utilisée uniquement comme modificateur adverbial du verbe mamit utin «il vente de l'est». La situation en cri des Plaines est sensiblement la même qu'en innu, car on y retrouve mâhihk mâmihk « aval, à l'est »; natamihk « amont, à l'ouest ».

Nos données contiennent aussi d'autres exemples d'orientations cardinales fondées sur des particularités hydrographiques locales. En cri de l'Est (dialecte du Nord) on peut, par exemple, utiliser la particule tâwich « au large » pour modifier le verbe uhtin «il vente d'une certaine direction». Le sens du syntagme verbal tâwich uhtin est alors « il vente du large (de la Baie James) » et, par extension, « il vente de l'ouest », puisque, dans ces communautés côtières de la Baie James et de la Baie d'Hudson, le large est à l'ouest. De plus, à Whapmagoostui, le verbe yâyâwâshtin " il vente de la côte » (avec la finale II -âshtin " ça souffle »), qui est dérivé de la particule yâyâw " grève, côte », signifie, par extension pragmatique, « il vente du sud-ouest », car, à cet endroit, la côte de la Baie d'Hudson est orientée SUD-OUEST/NORD-EST. Enfin, le verbe impersonnel umushâwâtin « il vente de la toundra (EST) » présente une finale -tin « venter » (II) ajoutée à une initiale umushâwâ- "stérile, dénudé » qui fait référence à la toundra forestière située à l'est de cette communauté côtière, c'est-à-dire dans l'intérieur des terres.

\subsection{Déité cardinaleléolienne}

Dans cette section, nous examinons les mots qui expriment la direction cardinale SUD, et montrons que les noms des déités cardinales et les directions cardinales dans lesquelles elles résident peuvent servir de source de lexicalisation. Les langues algonquiennes sont remarquablement uniformes quant à l'expression de la direction SUD, puisque plusieurs langues algonquiennes éloignées géographiquement et génétiquement font usage d'un réflexe de PA *sha·wanw « au, dans le sud »- qui se présente seul (cheyenne) ou suivi d'un locatif (cri de l'Est, abénaquis) - comme l'indiquent les exemples suivants :

Exemple 5. a. sóvóne (cheyenne, algonquien de l'Ouest) (Cheyenne Dictionary 2003-2014)

b. shâwanihch (cri de l'Est, dialecte du Sud-intérieur, algonquien du Centre)

c. zowanakik (abénaquis, algonquien de l'Est) (Day 1994)

La plus vieille occurrence d'un réflexe de PA *sha wanw dans les écrits des grammairiens-missionnaires se retrouve chez Fabvre (ibid., p. 48), qui note cha8annir8tin «Il uente du midy». Potier (1751, p. 154) ${ }^{8}$ rapporte Cha8ánônronnon en wendat du XVIII ${ }^{\mathrm{e}}$ siècle, qu'il traduit par «Cha8ánôns », un 
terme qui devait renvoyer à des gens originaires du sud de la zone des Grands Lacs ou de la rive sud du fleuve Saint-Laurent. C'est d'ailleurs la signification notée par Lemoine (1909) - câwanok « les gens du Sud» (NA) - pour l'algonquin du XIX ${ }^{\mathrm{e}}$ siècle.

La source de lexicalisation de ces particules est probablement très ancienne, car les langues de l'exemple 5 sont séparées par plusieurs milliers de kilomètres, étant admis qu'en dialectologie la forme linguistique qui présente la diffusion géographique la plus importante est souvent aussi plus conservatrice sémantiquement (MacKenzie 1980, p. 167). Selon Proulx (1986, p. 21), les réflexes de *sha·wanw auraient - en raison de l'opacité sémantique de cette proto-forme et de la distribution géographique répandue de ses réflexes - un niveau élevé de saillance dans les langues algonquiennes. Cependant, alors que Proulx voit dans la course du soleil la source de lexicalisation de *sha wanw, nous pensons, en accord avec les grammairiens-missionnaires, qu'il pourrait s'agir du nom propre de la déité méridionale (ou de sa demeure cardinale). Ce terme archaïque semble avoir subi un changement métonymique par contiguïté associant l'abstrait (déité ou direction méridionale) au concret (endroit où habite un peuple donné).

En effet, en interrogeant d'un peu plus près l'imposante mythologie mesquakie, recueillie par l'ethnologue amérindien William Jones au début du siècle dernier, il est clair que la direction SUD est associée à la loge des oiseaux-tonnerres - une direction aussi occupée par la déité méridionale. Jones écrit : "The name of the south wind is Cāwanaanwi, and $\mathrm{Ca} w a n^{\mathrm{a}}$ is the name of the great manitou of the south. He and the South Wind are friends. In the lodge of Cāwan ${ }^{\mathrm{a}}$ dwell the Thunderers, that go forth to guard the people " (1911, p. 212). Bloomfield signale dans sa grammaire du menominee (1962, p. 249) que sa.wanow «Southerner, Shawnee 9 " est dérivé du substantif sa·wanoh « in the south». Le premier terme présente un suffixe nominal d'agentivité -ow que l'on retrouve aussi dans wa.panow " member of the Morning Star cult». Pearson (1987, p. 189) abonde dans le même sens en soutenant que Šaawanwa est l'auto-ethnonyme au moyen duquel les Shawnee, un peuple qui habitait originellement le Sud-Est des ÉtatsUnis, se désigne.

Ces données de la mythologie mesquakie indiquent qu'il est possible que le nom propre d'un peuple ou d'une déité cardinale - résidant dans la direction habitée par le soleil du midi (zénith) et les oiseaux migrateurs - servent à nommer une portion du cadran cardinal. Cette source de lexicalisation est très peu mentionnée dans la littérature, mais Levinson (1996, p. 146) indique qu'il n'est pas rare qu'un nom de lieu important sur le plan symbolique ou spirituel devienne lexicalisé comme direction cardinale. Brauner (1998, p. 27) par exemple, montre qu'en maasai le terme pour SUD o'meroi signifie littéralement «combattre, guerre », alors que celui pour NORD kōpekob renvoie au territoire ancestral d'où sont originaires les Maasai. C'est que la migration de ce peuple s'est fait du nord au sud, ce dernier étant un territoire ennemi. Ailleurs encore, un 
(auto-)ethnonyme ethnique peut devenir lexicalisé comme direction cardinale (Mietzner et Pasch 2007, p. 12). Toutefois, dans l'état actuel de nos connaissances et en l'absence de documents anciens, il est difficile, voire impossible, de dire si ce sont les Shawnee ou les Mesquakie qui ont étendu un nom propre de déité (ou de personne s'adonnant à un culte particulier) à la direction cardinale où il loge, ou si c'est le substantif exprimant la déité méridionale qui a comme source de lexicalisation le nom d'une région restée dans la mémoire collective pour son importance symbolico-culturelle. Nous y reviendrons dans la section suivante.

\section{DU SYSTÈME ORIGINEL PROTO-ALGONQUIEN AUX INNOVATIONS LOCALES}

Maintenant que nous avons identifié et analysé les sources de lexicalisation (cycle solaire, direction du vent, cours des rivières, déité éolienne/cardinale) des termes de directions cardinales dans les dialectes de l'algonquien du Centre, il nous faut recomposer chacun des microsystèmes lexicaux à l'étude, afin d'en dégager les relations d'opposition morpholexicales. L'objectif de cette section est de tenter de cerner, d'une part, les causes et les conséquences du changement linguistique (innovation, tabou linguistique, chevauchement, remplacement) dans un champ lexical relativement abstrait et de vérifier, d'autre part, une hypothèse qui figure chez Swadesh (2006 [1971], p. 32), et qui fut reprise par Proulx (1986, 1988), et selon laquelle : « [...] while linguistic change is always slow, its rate of change varies with social, economic, political, and geographic factors; furthermore, some portions of language change less slowly than others ".

Deux questions nous serviront d'assise analytique :

1. Est-ce que les directions ou les places cardinales sont exprimées par des mots :

1a. appartenant au même microsystème d'opposition lexicale (ex. droite/ gauche, crépuscule/aube, amont/aval, haut/bas) ;

1b. n'appartenant pas au même microsystème d'opposition lexicale (ex. vent vs cycle solaire)?

2. Est-ce que les directions ou les places cardinales sont exprimées :

2a. par absence de marquage morphologique spécial, comme la conversion syntaxique (ex. en innu tshiuetin signifie « il vente du nord »[II], mais aussi « le nord $\gg[\mathrm{NI}])$;

2b. par ajout d'un suffixe du locatif (chiiwaatinuuhch « le nord » [NI], chiiwaatinuutaahch « au/vers le nord » [PT] [naskapi]) ;

2c. par ajout de marqueurs distincts sur un item lexical qui sert de point d'ancrage lexical? 
Il existe deux possibilités :

2c-i. le marqueur indique une opposition lexicale (ex. arctiquelantarctique) ;

2c-ii. le marqueur est un augmentatif (pâshtâ-) ou un diminutif (-ishîsh).

Dans ce qui suit nous allons étudier douze microsystèmes lexicaux en procédant d'OUEST en EST, c'est-à-dire en commençant par ceux qui ont la diffusion géographique la plus large - proto-algonquien (Figure 2); ojibwa (Figure 3) ; algonquin (Figure 4); cri de Moose (Figure 5); cri de l'Est (dialecte du Sud) (Figures 6a et 6b) ; cri des Plaines (Figure 7a) ; naskapi (Figures 8a et 8b) - pour nous pencher ensuite sur les dialectes qui ont innové en la matière : cri des Plaines (Figure 7b) ; cri de l'Est (dialecte du Nord) (Figure 10) et innu (Figure 11).

\subsection{Systèmes conservateurs (quatre termes archaïques)}

Nous avons vu, dans la section 3, qu'il existe, dans plusieurs dialectes et langues appartenant à l'algonquien du Centre, des termes de direction archaïques dont la source de lexicalisation est le cycle solaire, ou les noms de déités/directions cardinales. Trois termes sur quatre ont pu être reconstruits par les spécialistes du proto-algonquien, il s'agit de PA *sha·wanwi « au/le sud», de *wa.panwi «c'est

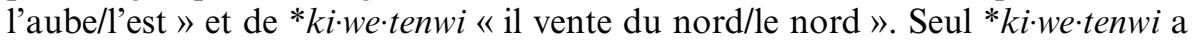
une distribution plus septentrionale dans le domaine algonquien, car les données provenant des autres langues algonquiennes montrent que *pepo $n w i$ « hiver » est probablement le terme archaïque pour désigner la direction NORD (Proulx 1986, p. 22) ; les deux autres ont des réflexes dans des langues algonquiennes séparées par des milliers de kilomètres comme le cheyenne, le mi'kmaq ou le naskapi. La Figure 2 introduit les termes pour le proto-algonquien. La proto-forme pour la direction OUEST reste inconnue à ce jour.

Dans les figures de cette section, les sources de lexicalisation sont indiquées par des petites capitales, quand celles-ci sont sans équivoque, et par des lettres minuscules, quand elles ne sont pas entièrement sûres comme c'est le cas pour SUD. De plus, le point d'interrogation (?) indique que la source de lexicalisation est inconnue, comme dans les Figures 3 et 4 par exemple.

Nous débutons avec les microsystèmes cardinaux de l'ojibwé et de l'algonquin (les termes de l'atikamekw sont similaires à ceux du cri de Moose) - deux dialectes situés à proximité de la zone d'occupation originelle des peuples algonquiens (Siebert 1967) - pour progresser vers la périphérie du continuum CINA (cri de l'Est, naskapi et innu), c'est-à-dire vers l'extrême est du Québec.

Les microsystèmes des Figures 3 et 4 sont probablement très anciens puisque trois termes sur quatre (NORD, EST, SUD) sont répandus sur de longues distances dans le domaine algonquien. Nous n'avons pu déterminer la source de lexicalisation des particules pour OUEST en ojibwé ou en algonquin. Toutefois, on peut 


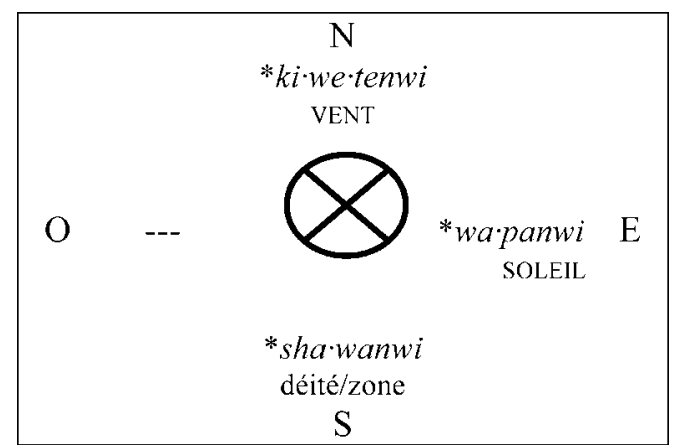

FIG. 2 - Proto-algonquien (Hewson 1993).

FIG. 3 - Ojibwé (Nyholm et Nichols 1995).
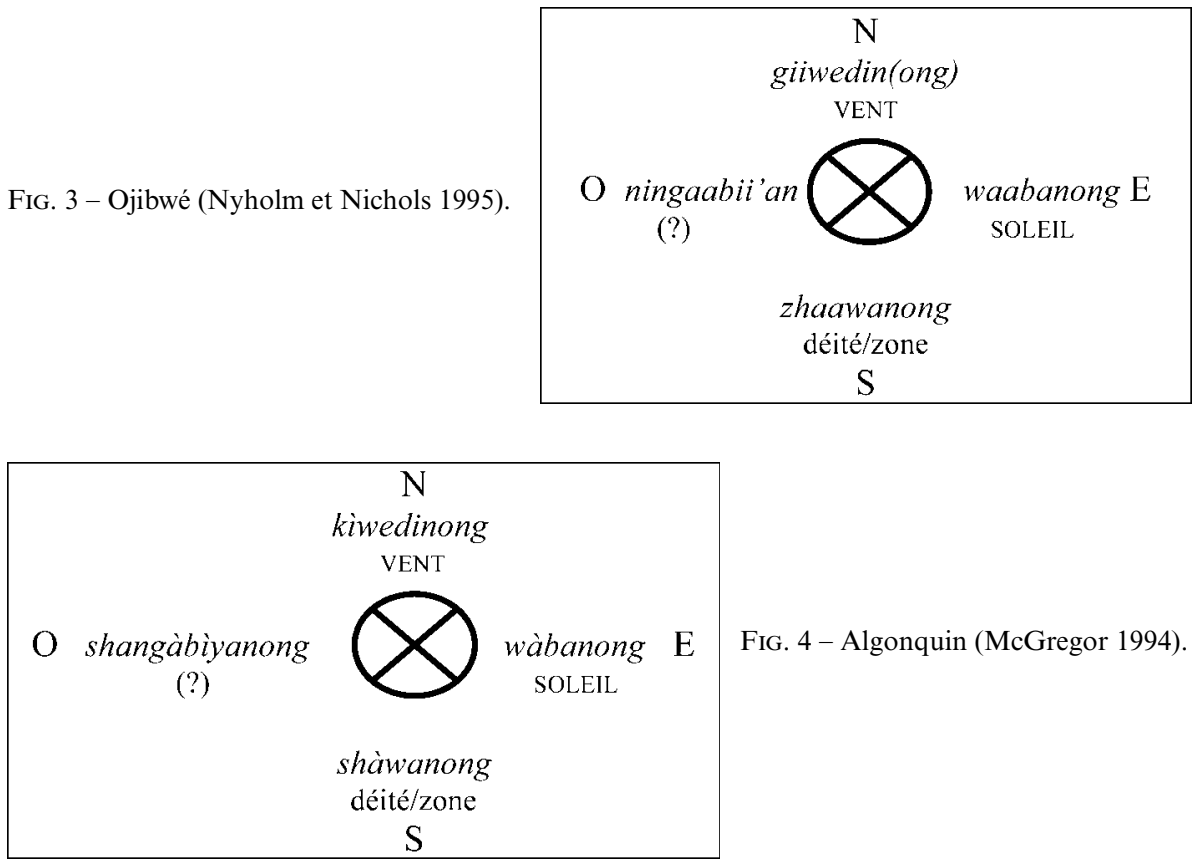

FIG. 5 - Cri de Moose (Brousseau et Collette 2014).

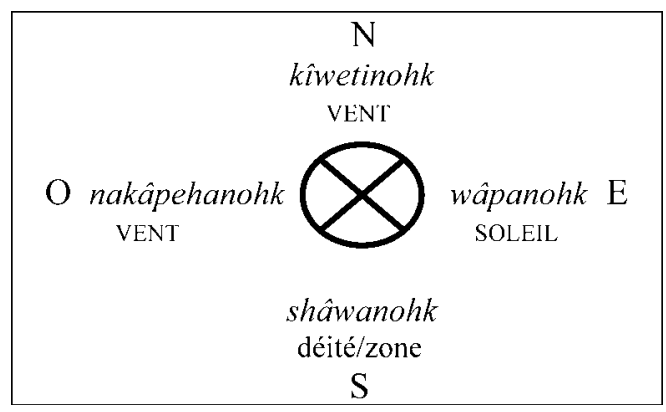


FIG. 6a - Cri de l'Est (dialecte du Sudintérieur) (Neeposh et al. 2004)
O nakâpehanûtâhch VENT
$\mathrm{N}$

chîwetinûtâhch

VENT

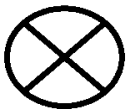

wâpanûtâhch $\mathrm{E}$ SOLEII.

sôwanihch

déité/zone

$\mathrm{S}$

O pahkismohâhk

Fig. 7a - Cri des Plaines (Wolvengrey 2001).

FIG. 8a - Naskapi (MacKenzie et Jancewicz 1994).

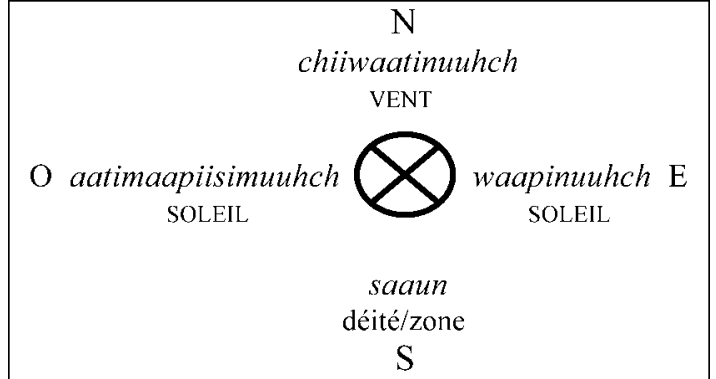

$\mathrm{N}$

chiiwaatinuuhch

VENT

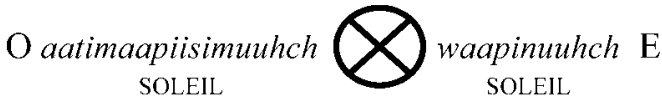

FIG. 8b - Naskapi (innovation)

(MacKenzie et Jancewicz 1994).

\section{piisimuuhch}

SOLEIL

$\mathrm{S}$ 


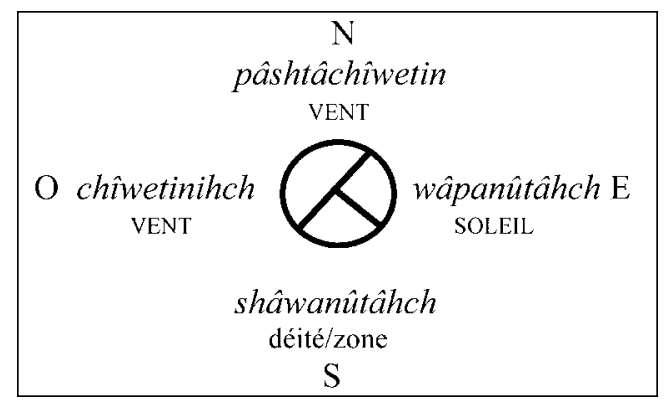

FIG. 6b - Cri de l'Est (dialecte du Sudintérieur ; données de l'auteur, locuteur de Mistissini).

FIG. 7b - Cri des Plaines (Wolvengrey 2001).
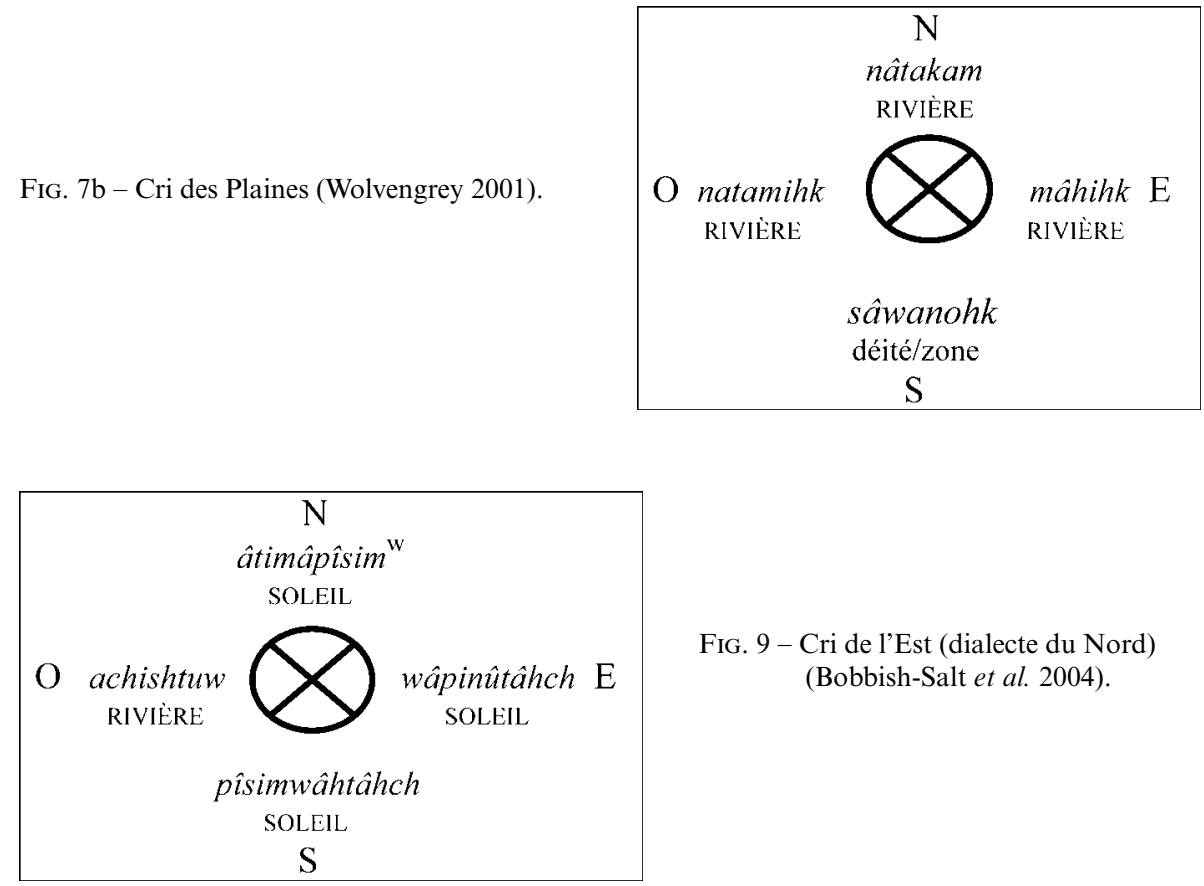

FIG. 9 - Cri de l'Est (dialecte du Nord) (Bobbish-Salt et al. 2004).

Fig. 10 - Innu (www.innu-aimun.ca).

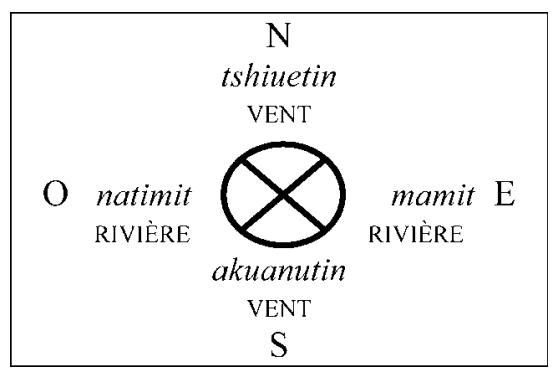


penser qu'elles sont archaïques puisqu'elles sont opaques sémantiquement et que les réflexes modernes présentent des variations phonologiques.

Sont représentés, dans les Figures 5 et 6a, les microsystèmes lexicaux des dialectes cris correspondant à ceux de l'ojibwé et de l'algonquin. En ce qui concerne la direction OUEST, nous avons montré dans la section 3.2 que la source de lexicalisation était, en synchronie, un verbe impersonnel éolien nakâpehan« venter de l'ouest » nakâpehanû-tâhch « du côté de l'ouest » (PT). L'étymologie de ce terme reste inconnue pour l'instant.

Le cri de l'Ouest, l'algonquin et l'ojibwé ont des réflexes de PA *pankihshimowa « il (soleil) se couche » (IA) (de *panki- « tomber, échapper » + *-hshim« étendre, aboutir dans telle position »). Notons par exemple epangishimodj en algonquin, ou encore pahkisimohk en cri des Plaines (Figure 7a). Sur le plan morphologique, ces particules sont des locutions issues d'un verbe conjonctif lexicalisé comme particule adverbiale de lieu, même si, à l'origine, on peut penser qu'elles ne faisaient qu'un avec le nom de l'esprit suprême Epingishmook, intendant du royaume de l'Ouest et des morts. La Figure 7a présente un des microsystèmes où il est fait usage de ce verbe lexicalisé.

Comme l'indiquent les Figures 2, 3, 4, 5, 6a et 7a, seuls les termes exprimant la direction OUEST dans les langues et dialectes occidentaux de notre échantillon présentent une variation (vent, soleil) dans les sources de lexicalisation. Comment expliquer la présence de cette variation dans ces microsystèmes lexicaux archaïques? Dans la section 3.2, nous avons vu que les données linguistiques issues de la mythologie n'éclaircissaient que très peu l'étymologie comparée des termes pour OUEST. Toutefois, le symbolisme et les connotations culturelles que véhicule la mythologie - en tant que littérature orale ancienne - permettent de dégager certains éléments de réponse. En effet, dans l'enseignement traditionnel de certains groupes algonquiens, la direction OUEST est reliée à la fin du cycle quotidien du soleil, au monde des morts, à l'hiver et aux esprits éoliens masculins. Il est possible que la présence d'une variation phonologique (par exemple $/ \mathrm{n} / \mathrm{et} / \mathrm{sh} / \mathrm{des}$ mots pour OUEST des Figures 3 et 4) et lexicale (prenant la forme d'un chevauchement) fut motivée, à l'origine, par des règles culturelles d'évitement qui se sont ensuite concrétisées verbalement par des tabous linguistiques ${ }^{10}$; ceux-ci sont reconnus en linguistique historique comme étant des vecteurs de changement sémantique (Hock 1991, pp. 294, 303). On peut supposer que les transformations sociétales provoquées par la traite des fourrures et l'arrivée des missionnaires ont eu un impact sur la préservation ou non de certaines des connotations culturelles sous-jacentes au système de direction cardinale ${ }^{11}$.

Comme nous allons le voir plus loin, il y a, dans les dialectes cris, un chevauchement, en synchronie, entre un système d'opposition lexicale fondé sur le tracé quotidien du soleil (Figure 7a) et un autre fondé sur des particularités hydrographiques locales (Figure 7b). 


\subsection{Systèmes localisés (trois termes archaïques)}

Tournons-nous maintenant du côté des microsystèmes cardinaux à trois termes archaïques. Comme en cri de l'Est (dialecte du Sud-intérieur) et en cri des Plaines, le naskapi fonctionne avec deux systèmes qui coexistent en synchronie. L'un a le cycle du soleil et le vent du nord comme sources de lexicalisation, alors que l'autre présente des réflexes des termes archaïques, sauf pour la direction OUEST qui est clairement une innovation locale. C'est en naskapi que la différence de sens entre les locatifs $-^{\wedge} h c h$ et $-^{\wedge}$ taahch est la plus claire : le premier est un substantif locatif absolu (waapinuuhch «l'est »), alors que l'autre est une particule locative directionnelle indiquant l'idée de mouvement vers une direction (waapinuuhtaahch "vers l'est »). La plupart des termes de direction du naskapi peuvent prendre l'un ou l'autre de ces suffixes. Les Figures $8 \mathrm{a}$ et $8 \mathrm{~b}$ présentent les données du naskapi.

Dans la Figure 8b, on remarquera que : a. le chevauchement lexical affecte seulement la direction SUD puisque le terme archaïque alterne avec un autre terme ayant le soleil comme source de lexicalisation ; et b. l'initiale aatim- «à l'opposé » dans aatimaapiisimuuhch « l'ouest » (NI) signifie littéralement « à l'opposé de l'est (du soleil levant)» et non pas « à l'opposé du sud (zénith)» comme pourrait le suggérer l'analyse morphologique ${ }^{12}$.

Enfin, le cri de l'Est (dialecte du Sud) présente un microsystème cardinal où la forme chîwetin- indique non pas un vent du nord mais bien un vent d'ouest. La Figure 6b nous provient du cri de l'Est (dialecte du Sud-intérieur) et ce système coexiste avec le celui présenté dans la section 4.1.

La Figure 6b présente une innovation unique sur le plan lexical puisque l'on est en présence d'un seul lexème verbal chîwetin- avec un sens qui diffère de la signification « il vente du nord » attestée jusqu'à présent, ce qu'indique le découpage asymétrique dans la Figure $6 \mathrm{~b}$. Nous allons tenter de comprendre à quoi est dû cet élargissement de sens.

Tout d'abord, ce découpage particulier du cadran cardinal éolien trouve écho dans les sources missionnaires de la fin du XVII ${ }^{\mathrm{e}}$ siècle. En effet, Silvy (1974 [1678], p. 54) note ki8etin "nord-ouest, vent froid », alors que Fabvre (1970 [1695]) présente, à côté de ki8êtin "nord8ast », le dérivé ki8êtinask8an « Les nuées en uiennent » (où -ask8an correspond à la médiane -askw « nuage » en cri de l'Est), mais aussi pit8tchi8etin « vent nord, nord » (ibid., p. 314). Ce dernier terme n'est pas attesté en cri, ni en innu, et aucun document ancien ne présente, à notre connaissance, de verbe pour «il vente de l'ouest/l'ouest», mis à part ki8êtin « nord8ast». Certains dialectes actuels sont restés proches de la situation qui prévalait il y a trois cents ans. En cri de l'Est (dialecte du Nord) et en naskapi par exemple, le lexème chîwâtin- a un large spectre dans le cadran cardinal et les oppositions lexicales de l'axe NORD-OUEST sont obtenues grâce à l'ajout d'une initiale pâshtâ- « au-delà, de l'autre côté ». Afin de se donner une idée du spectre 
lexical couvert par le lexème chîwâtin-, examinons les verbes éoliens impersonnels (II) tirés de trois sous-dialectes voisins, soit le cri de l'Est (dialecte du Nord parlé à Chisasibi et à Whapmagoostui) et le naskapi. On notera que le microsystème des verbes éoliens de Whapmagoostui (cri de l'Est, dialecte du Nord) est parallèle à celui des directions cardinales du cri du Sud-Intérieur (Annexe 2, Tableau 2).

\begin{tabular}{|c|c|c|}
\hline $\begin{array}{c}\text { Cri de l'Est } \\
\text { (dialecte du Nord, Chisasibi) } \\
\text { [Bobbish-Salt et al. } 2004]\end{array}$ & $\begin{array}{c}\text { Naskapi } \\
\text { (Kawawachikamach) } \\
\text { [Mackenzie et Jancewicz 1994] }\end{array}$ & $\begin{array}{c}\text { Cri de l'Est } \\
\text { (dialecte du Nord Whapmagoostui) } \\
\text { [données de l'auteur] }\end{array}$ \\
\hline $\begin{array}{l}\text { chîâtin } \\
\text { il vente du nord } \\
\text { pâshtâchîwâtin } \\
\text { il vente du nord-ouest } \\
\text { tâwich uhtin } \\
\text { il vente du large (de l'ouest) }\end{array}$ & $\begin{array}{l}\text { chiiwaatin } \\
\text { il vente du nord } \\
\text { paashtaachiiwaatin } \\
\text { il vente du nord-ouest }\end{array}$ & $\begin{array}{l}\text { pâshtâchîwatin } \\
\text { il vente du nord } \\
\text { chîâtinisis } \\
\text { le nord-ouest } \\
\text { chiwâtin } \\
\text { il vente de l'ouest }\end{array}$ \\
\hline
\end{tabular}

FIG. 11 - Variations des termes basés sur le lexème chîwâtin.

Ces données montrent que la forme chîwâtin- (ou chiiwaatin-) exprime des lexèmes « nord » et « ouest » qui diffèrent selon les dialectes et les microsystèmes particuliers. Toutefois, pour donner encore plus de sens à cette donnée, il importe de se rappeler que la direction du vent n'est en rien un point de repère absolu et régulier, comme l'est le Nord magnétique. En effet, un vent peut prendre de la puissance, tourner ou mourir, et ce qui semble être un vent du nord dans un lieu peut être ressenti comme un vent d'ouest ou du nord-ouest ailleurs. L'argument avancé par certains anthropologues (Feit 1978, pp. 289 sq. ; Tanner 1979, pp. 95 $s q$. ; Sukran Tipi, com. pers. 2012) est que les vents d'ouest et du nord appartiennent à la même classe de vents, c'est-à-dire à des vents d'hiver froids, secs et dominants ${ }^{13}$. Ainsi, il semble possible d'avancer que l'utilisation d'un seul item lexical polysémique comme point d'ancrage lexical soit motivée par les conditions atmosphériques spécifiques qu'apportent les vents provenant des directions NORD, NORD-OUEST et OUEST. Que celles-ci soient ressenties et perçues comme relevant du même domaine est tout à fait cohérent avec le découpage de l'espace domestique qui se fait selon l'axe NORD-EST/SUD-OUEST : le SUD et l'EST constituent un espace féminin (chaleur, été, naissance), alors que le NORD et l'ouEsT sont des espaces masculins (froid, hiver, mort).

S'il paraît évident que nous avons affaire à une innovation localisée, nous avançons qu'elle n'est en rien récente puisque, d'une part, ces variations dans le spectre de chîwâtin/chîwetin furent notées par les missionnaires au XVII ${ }^{\mathrm{e}}$ siècle, mais que, d'autre part, on peut voir dans certaines variations régionales $\mathrm{du}$ cri de l'Est une sorte de calque des connotations véhiculées par la mythologie au sein même des oppositions morphologiques. C'est-à-dire que le diminutif -shîsh/-sis de chîwetinishîsh/chîwâtinisis peut revêtir, pour certains locuteurs, une 
signification strictement atmosphérique et signifier « vent du (nord-)ouest 》 (NI) ou encore « vent léger du nord » (NI). Pour d'autres locuteurs chîwetinishîsh a une connotation culturelle au sens où le diminutif rappelle le duel symbolique opposant la grandeur de l'aîné ${ }^{14}$ (déité éolienne du nord) à la fougue du cadet (déité éolienne de l'ouest) ${ }^{15}$. D'ailleurs, un de nos informateurs de Mistissini (un homme dans la cinquantaine) considérait chîwetinishîsh comme le «little soldier of chîwetin, the northern wind ». Dans les transformations socio-culturelles provoquées par la sédentarisation et l'évangélisation, ces termes à connotations animistes (lesquelles ne sont en rien senties par tous les locuteurs modernes) ont plus de chance d'être consciemment remplacés par des termes plus neutres ayant comme source de lexicalisation autre chose qu'un vent ou une déité.

\subsection{Systèmes innovants (un terme archaïque)}

Déplaçons-nous vers la périphérie du continuum CINA (c'est-à-dire hors du pourtour des Grands Lacs) pour étudier le cri des Plaines, le cri de l'Est (dialecte du Nord) et l'innu. Nous avons classé ces microsystèmes ensemble puisqu'ils présentent, chacun, un seul des termes archaïques. Ces trois microsystèmes sont ceux pour lesquels la notion de direction cardinale s'applique avec le plus de difficulté puisque le point de repère est atteignable et donc relatif. C'est la raison pour laquelle nous préférons parler de « place cardinale » et non de « direction cardinale $"$.

Les deux microsystèmes précédents ont innové puisqu'ils ont des particules adverbiales de lieu (amont/aval) pour référer à des directions sur l'axe EST-OUEST. Comme l'indique la Figure $7 \mathrm{~b}$, le microsystème novateur du cri des Plaines a le cours des grandes rivières de l'Alberta et de la Saskatchewan comme source de lexicalisation. On imagine que la lexicalisation de ces particules adverbiales de lieu hydrographique a été facilitée par la sédentarisation progressive et l'augmentation des brigades de canots transportant biens et fourrures sur un immense réseau fluvial.

Penchons-nous maintenant sur la Figure 9. On voit que les directions cardinales NORD et SUD sont exprimées par des mots ayant le cycle solaire comme source de lexicalisation. Sur le plan morphologique, c'est l'initiale âtim- " opposé à » qui fonde l'opposition lexicale pîsimwâhtâhch «le sud, au sud » /âtimâpîsim ${ }^{w}$ " le nord, au nord », à la manière du couple français « arctique/ant-arctique ». En cri toutefois, c'est la direction cardinale SUD qui est le point d'ancrage de l'opposition. En naskapi aatimaa- «à l'opposé » dans aatimaapiisimuutaahch «à l'ouest » (PT) réfère uniquement à l'opposé du soleil levant, et non pas à piisimuutaahch « au sud » (NI). Toutefois, dans la Figure 9, la direction OUEST est exprimée par la particule adverbiale de lieu achishtuw. Selon Neacappo (2012, p. 88, note 35$)$, la signification littérale de cette particule renverrait à la position descendante du soleil en fin de journée. Si l'hypothèse du cycle solaire s'avérait 
juste, alors ce système serait le seul exemple de notre échantillon à avoir le cycle du soleil comme source de lexicalisation pour les deux axes NORD-SUD et EST-OUEST. Nous proposons ici une analyse différente et avançons que l'initiale achisht(/achit/) achihch-indique une position renversée dans laquelle le haut est sous le bas. Cette signification se retrouve dans les mots achishtikuhtin «ça flotte à l'envers » (II); achichihtin " c'est placé à l'envers » (II); achishtiwâw « la pente (descendante) cache le rapide en aval» (II). Si la pente décrite par ce dernier verbe est réellement celle d'une rivière, alors il est probable qu'elle fut étendue pour référer à la direction OUEST puisque, dans le bassin hydrographique de la Baie James, la plupart des grandes rivières de navigation qui permettent d'atteindre la côte, où étaient situés les postes de traite de la Compagnie de la Baie d'Hudson jusqu'au siècle dernier, coulent d'EST en OUEST. Deux autres faits viennent corroborer la thèse des particularités hydrographiques comme source de lexicalisation. Premièrement, dans certaines variétés du cri de l'Est (dialecte Sud-intérieur), la particule achishtuw réfère non pas à l'OUEST mais au NORD (Mistissini) ou même au NORD-OUEST ou à l'OUEST (Nemaska) (com. pers. avec Kevin Brousseau) ce qui indique que achishtuw ne refère peut-être plus au cycle solaire mais bien au cours des rivières. Deuxièmement, à Chisasibi nikâpâhân " il vente de l'ouest » (II) coexiste avec tâwich ûhtin " il vente du large » alors que achishtuw ûhtin « il vente de l'ouest » ne semble pas utilisé.

Enfin, dans le système presque entièrement rénové de l'innu, seul le terme archaïque tshiuetin «vent du nord (II)/Nord (NI)» subsiste encore comme l'indique la Figure 10.

Le verbe akualutin « il vente du sud» (II)/« Sud» (NI) est formé par analogie avec le terme tshiuetin « il vente du nord » (II)/« le Nord» (NI), et il a une saveur pragmatique toute spéciale puisqu'il renvoie à un "vent» (-lutin) venant de l'« autre rive » (akua-) - en l'occurrence, la rive sud du fleuve Saint-Laurent qui fait échouer les bateaux sur la rive nord. Les deux autres orientations cardinales natimit et mamit sont des extensions localisées (ou cas de synesthésie) des particules adverbiales de lieu allant du concret amont $\leftrightarrow$ aval à l'abstrait OUEST $\leftrightarrow$ EST. Le fait que ces innovations se soient répandues dans des régions où les grandes rivières navigables coulent du nord au sud indique très clairement que le changement s'est opéré d'abord dans la Haute-Côte-Nord (là où le flot des rivières est orienté OUEST-EST, comme pour la rivière Saguenay) pour être adopté, sous forme lexicalisée, dans la Basse-Côte-Nord du Saint-Laurent.

$\mathrm{Au}$ final, les termes des microsystèmes cardinaux du cri des Plaines (Figure 7b), du cri de l'Est (dialecte du Nord ; Figure 9) et de l'innu (Figure 10) illustrent avec précision la thèse de Proulx (1986, p. 22), à savoir que, dans les sociétés traditionnelles, les contours géographiques et hydrographiques d'une région, et les activités qui y sont reliées (comme le retour au poste de traite situé en aval à la débâcle des glaces), sont plus saillants pour les locuteurs que ne le sont les directions cardinales en elles-mêmes. Nous avons tenté de montrer que la 
nature et les fonctions spirituelles et socio-économiques de certains référents motivent, dans une certaine mesure, le renouvellement et le remplacement des termes de directions. Par exemple, le point commun entre les microsystèmes à un terme archaïque est qu'ils sont utilisés dans des communautés linguistiques situées sur les côtes et dans la périphérie du continuum CINA. Il est évident que la transformation de ces microsystèmes, au moyen d'extensions pragmatiques localisées, n'a pas pu se faire avant que la (semi-)sédentarisation autour d'un poste de traite devienne la norme socio-économique dans la région, c'est-à-dire vers la fin du XVIII ${ }^{\mathrm{e}}$ siècle pour les Cris de l'Est de la Baie James (surtout les côtiers), un siècle avant pour les Innu, et beaucoup plus tard pour les Cris des Plaines. Toutefois, ce n'est pas la cohabitation stricto sensu avec des EuroCanadiens (anglophones pour les uns et francophones pour l'autre) qui a provoqué le remplacement lexical de certains mots archaïques, mais bien le fait que les avals des grandes rivières de navigation - là où les marchands de fourrures et missionnaires avaient élu domicile - sont devenus, avec le temps, des lieux de plus en plus importants pour les activités socio-économiques des chasseurscueilleurs du subarctique canadien, contribuant ainsi à la transformation des microsystèmes cardinaux en périphérie du continuum CINA. D'autres recherches en lexicographie pourront nous permettre de mieux comprendre l'influence des grammairiens-missionnaires dans la formation des termes de directions et dans le remplacement des termes à forte connotation animiste, c'est-à-dire ceux qui ont des noms de déités éoliennes/cardinales comme source de lexicalisation.

\section{Conclusion}

Cet article a poursuivi deux objectifs complémentaires, lesquels consistaient à recenser, dans le cadre d'une étymologie comparée des langues et dialectes appartenant à la branche géographique de l'algonquien du Centre, les sources de lexicalisation des termes de directions cardinales ainsi qu'à dégager certaines tendances en ce qui a trait aux causes et aux conséquences du changement linguistique. Nous l'avons fait en sortant du domaine strictement linguistique afin d'embrasser des données symboliques et culturelles tirées de la mythologie. Dans la section 1, nous avons délimité les concepts de « point », « direction » et «place» cardinale et avons montré que la notion de quadricardinalité est ancienne puisqu'elle est fortement ancrée dans la mythologie, dans les enseignements traditionnels et dans la répartition de l'espace domestique. Dans la section 2, nous avons introduit certains éléments de morphologie des langues algonquiennes. Dans la section 3, nous avons identifié les sources de lexicalisation (cycle solaire, direction des vents, cours des rivières, déité éolienne/cardinale) des mots servant à exprimer les directions et les places cardinales dans une dizaine de dialectes et langues appartenant à la branche géographique de l'algonquien du 
Centre. Enfin, dans la section 4, nous avons analysé douze microsystèmes cardinaux en suivant un calcul précis de la diversité morpholexicale pour ce champ défini.

Notre description des termes de directions et des places cardinales (section 3) a mis au jour un fait relativement peu développé dans la littérature, à savoir que certains termes archaïques semblent avoir eu, à l'origine, un nom de déité éolienne/cardinale ou la zone où elle loge (sud) comme source de lexicalisation. Cela n'est en rien surprenant compte tenu de l'importance de ce type de déités dans la cosmologie algonquienne. Évidemment, en synchronie, ce lien étymologique n'est plus perçu comme tel. Notre analyse a aussi montré que les microsystèmes à quatre termes archaïques (sous-section 4.1) sont répandus sur de grandes distances géographiques, mais tout spécialement dans la zone centrale du continuum CINA (cris des Plaines, de Moose et de l'Est), en algonquin et en ojibwé, et qu'ils sont plus conservateurs sur le plan sémantique, ce qui n'est pas le cas des autres microsystèmes utilisés dans les régions limitrophes du continuum CINA. En outre, nous avons montré que la plupart des remplacements lexicaux sont survenus dans la partie orientale du continuum CINA (atikamekw, cri de Moose et de l'Est), alors que les chevauchements lexicaux sont plus communs dans les dialectes cris. L'analyse des causes et des conséquences du changement linguistique nous a permis de mettre au jour les tendances suivantes :

- Les termes opaques ayant comme source de lexicalisation des déités éoliennes/cardinales sont remplacés par des termes sémantiquement transparents, et jamais le contraire. Toutefois, certains de mes informateurs bilingues parlant le cri de l'Est (dialecte du Sud) affirment que le terme archaïque shâwan(ihch) «au/dans le sud» (PT) est un emprunt à l'anglais South. MacKenzie et Jancewicz (1994) ont noté la même chose pour le naskapi saaun « le Sud ». L'étymologie populaire ou même la remotivation joueraient donc un certain rôle dans le remplacement lexical dans la mesure où les locuteurs bilingues peuvent délaisser délibérément un terme archaïque comme shâwan (ihch) (faussement réinterprété comme un anglicisme) au profit d'une particule entièrement transparente comme pîsimwehtâhch « au/dans le sud».

- La synesthésie (extension sémantique qui va du concret à l'abstrait) et l'élargissement de sens sont des conséquences observables du changement dans le vocabulaire des termes de directions.

- Les données du cri de l'Est (dialecte du Nord), du naskapi et de l'innu indiquent que le sens du remplacement des termes archaïques se fait selon l'échelle de lexicalisation suivante: (?, source inconnue pour «l'ouest») > DÉITÉ/zone > VENT > SOLEIL > RIVIÈRE. C'est-à-dire que le remplacement lexical affecte plus spécialement les mots qui conservent, de manière ouverte ou cryptique, des références ou des connotations en rapport avec la cosmologie traditionnelle. C'est pourquoi il n'existe pas de nouveau terme de direction cardinale qui a le nom d'une déité éolienne/cardinale comme source de lexicalisation. 
Dans les exemples suivants, le terme qui vient remplacer le mot archaïque tombé en désuétude est toujours plus à droite de celui-ci dans l'échelle :

$\begin{array}{lll}\text { remplacement lexical }(\rightarrow) & \text { dialectes } \\ \begin{array}{ll}\text { Termes archä̈ques } \\ \text { déité/zone }\end{array} & \rightarrow \text { vent }_{\text {sud }} & \text { innu } \\ (?), \text { soleil } & \rightarrow \text { rivière }_{\text {ouest/est }} & \text { innu } \\ (?), \text { vent } & \rightarrow \text { soleil }_{\text {ouest }} & \text { naskapi } \\ \text { vent } & \rightarrow \text { soleil }_{\text {nord }} & \text { cri de l'Est (dialecte du Nord, Whapmagoostui) } \\ (?), \text { vent } & \rightarrow \text { rivière }_{\text {ouest }} & \text { cri de l'Est (dialecte du Nord, Chisasibi, } \\ & & \text { Whapmagoostui) } \\ \text { chevauchement lexical }(\sim) & \text { dialectes } \\ \text { déité/zone } & \sim \text { soleil }_{\text {sud }} & \text { naskapi, cri de l'Est (dialecte du Sud-intérieur) } \\ (?), \text { vent } & \sim \text { vent }_{\text {ouest }} & \text { cri de l'Est (dialecte du Sud-intérieur) } \\ \text { vent } & \sim \text { rivière }_{\text {ouest }} & \text { cri de l'Est (dialecte du Sud-intérieur) } \\ \text { vent } & \sim \text { soleil }_{\text {nord }} & \text { cri de l'Est (dialecte du Sud-intérieur) } \\ \text { vent } & \sim \text { soleil }_{\text {sud/nord }} & \begin{array}{l}\text { cri de l'Est (dialecte du Nord, Chisasibi, } \\ \text { Whapmagoostui) }\end{array} \\ (?) & \sim \text { soleil }_{\text {ouest }} & \text { cri des Plaines, ojibwé } \\ \text { soleil } & \sim \text { soleil }_{\text {est/sud }} & \text { cri des Plaines }\end{array}$

Pour terminer, notre analyse a aussi montré que l'innovation lexicale peut être motivée par des facteurs extralinguistiques. En effet, si un nouveau terme est créé, il y a de fortes chances pour que son point de référence soit une particularité géographique relative - qui a acquis une importance socio-économique au cours de l'histoire - comme le cours des grandes rivières. Cette observation va à l'encontre de l'une des tendances relevées par Brown (1983, pp. 142 sq.) et qui prédit que, si un système de directions cardinales est créé, il aura le cycle du soleil comme source de lexicalisation. Les données des dialectes situés en périphérie du continuum CINA, comme l'innu ou le cri de l'Est (dialecte du Nord), indiquent exactement le contraire puisqu'on a le vent et/ou le cours des rivières comme sources de lexicalisation pour les termes de formation récente. Au final, le chevauchement des microsystèmes cardinaux (cris des Plaines et de l'Est) et le remplacement de l'un par l'autre (innu) dans les dialectes limitrophes sont riches d'enseignement puisqu'ils nous permettent de valider l'hypothèse de Proulx (1986, p. 22), à savoir que, dans les sociétés traditionnelles, le référent des termes de directions (RIVIÈRE, SOLEIL) est souvent beaucoup plus saillant que le terme lui-même, et que certains changements économiques, sociaux, culturels ou 
religieux, provoqués par la présence des personnes (comme les missionnaires) et des institutions de la société industrielle dominante, peuvent exacerber, voire accélérer, le remplacement des termes dans ce champ lexical précis. *

* Manuscrit reçu en mai 2014, accepté pour publication en octobre 2014. 


\section{AnNeXe 1}

L'annexe 1 contient les sources bibliographiques utilisées dans le présent article.

\begin{tabular}{|l|l|l|}
\hline Dialectes/langues & \multicolumn{1}{|c|}{ Sources } & Codes \\
\hline $\begin{array}{l}\text { cri des Plaines } \\
\text { (dialecte du Sud) }\end{array}$ & $\begin{array}{l}\text { Lacombe 1874; Wolfart et Ahenakew 1998; } \\
\text { Wolvengrey 2001; www.atlas-ling.ca }\end{array}$ & CRP \\
\hline ojibwé & $\begin{array}{l}\text { Nichols et Nyholm 1995; The Ojibwe People's } \\
\text { Dictionary [www.ojibwe.lib.umn.edu] }\end{array}$ & OJB \\
\hline cri de Moose & Brousseau et Collette 2014 & CRM \\
\hline algonquin & McGregor 1994 & ALG \\
\hline atikamekw & Béland 1978; Dinnison 1999 & ATK \\
\hline $\begin{array}{l}\text { cri de l'Est } \\
\text { (dialecte du Sud-intérieur) }\end{array}$ & $\begin{array}{l}\text { Neeposh } \text { et al. 2004 ; données personnelles de } \\
\text { l'auteur }{ }^{16} \text {; com. pers. avec Kevin Brousseau }\end{array}$ & CRS \\
\hline $\begin{array}{l}\text { cri de l'Est } \\
\text { (dialecte du Nord) }\end{array}$ & $\begin{array}{l}\text { Bobbish-Salt } \text { et al. } 2004 \text { (Chisasibi) ; Collette 2014 } \\
\text { (Whapmagoostui) }\end{array}$ & CRN \\
\hline naskapi de l'Ouest & MacKenzie et Jancewicz 1994 & NSK \\
\hline illu de Pessamit & Drapeau 1991; www.innu-aimun.ca & ILL \\
\hline innu de la Basse-Côte-nord & McNulty et Basile 1981; www.innu-aimun.ca & INN \\
\hline cri des Bois & Com. pers. avec Solomon Ratt & CRB \\
\hline
\end{tabular}




\section{Annexe 2}

Dans les tableaux suivants, la présentation des données se fait d'est en ouest, les données du cri des Plaines et du Mesquakie sont donc toujours présentées en dernier.

\begin{tabular}{|c|c|c|c|}
\hline $\begin{array}{l}\text { Dialectes } \\
\text { /langues }\end{array}$ & $\begin{array}{c}\text { Particule (PT) / Substantifs } \\
\text { inanimés (NI) }\end{array}$ & $\begin{array}{c}\text { Découpe } \\
\text { morphologique }\end{array}$ & Gloses \\
\hline NSK & $\begin{array}{l}\text { waapinuuhch }(\mathrm{NI})(\mathrm{E}) \sim \\
\text { waapinuutaahch }(\mathrm{PT}) \\
\text { piisimuutaahch }(\mathrm{PT})(\mathrm{S}) \\
\text { aatimaapiisimuuhch }(\mathrm{NI})(\mathrm{O}) \sim \\
\text { aatimaapiisimuutaahch }(\mathrm{NI})(\mathrm{O})\end{array}$ & $\begin{array}{l}\text { /wâpinw-^hch/ } \\
\text { /wâpinw-^tâhch/ } \\
\text { /pîsimw-^^âhch/ } \\
\text { /âtim-âpîsimw-^hch/ } \\
\text { /âtim-âpîsimw-^tâhch/ }\end{array}$ & $\begin{array}{l}\text { être.aube-LOC } \\
\text { être.aube-LOC } \\
\text { soleil(levant)-LOC } \\
\text { opposé-soleil-LOC } \\
\text { opposé-soleil(levant)-LOC }\end{array}$ \\
\hline $\begin{array}{c}\text { CRN } \\
\text { (Chisasibi, } \\
\text { Whapmagoostui) }\end{array}$ & $\begin{array}{l}\text { wâpinûtâhch }(\mathrm{PT})(\mathrm{E}) \\
\text { pîsimwâhtâhch }(\mathrm{PT})(\mathrm{S}) \\
\text { âtimâpîsimwâhtâhch }(\mathrm{PT})(\mathrm{N}) \\
\sim \text { âtimâpîsimw }(\mathrm{PT})(\mathrm{N})\end{array}$ & $\begin{array}{l}\text { /wâpinw-^tâhch/ } \\
\text { /pîsimw-âhtâhch/ } \\
\text { /âtim-âpîsimw-âhtâhch/ } \\
\text { /âtim-âpîsimw/ }\end{array}$ & $\begin{array}{l}\text { être.aube-LOC } \\
\text { soleil-LOC } \\
\text { opposé-soleil-LOC } \\
\text { opposé-soleil }\end{array}$ \\
\hline $\begin{array}{c}\text { CRS } \\
\text { (Mistissini) }\end{array}$ & $\begin{array}{l}\text { wâpanûtâhch }(\mathrm{PT})(\mathrm{E}) \\
\text { pîsimwehtâhch }(\mathrm{PT})(\mathrm{S})\end{array}$ & $\begin{array}{l}\text { /wâpanw-^tâhch/ } \\
\text { /pîsimw-ehtâhc/ }\end{array}$ & $\begin{array}{l}\text { être.aube-LOC } \\
\text { soleil(midi)-LOC }\end{array}$ \\
\hline CRM & $\begin{array}{l}\text { wâpanohk }(\mathrm{NI})(\mathrm{E}) \\
\text { wâpanôtâhk }(\mathrm{NI})(\mathrm{E})\end{array}$ & $\begin{array}{l}\text { /wâpanw-^hk/ } \\
\text { /wâpanw-^tâhk/ }\end{array}$ & $\begin{array}{l}\text { être.aube-LOC } \\
\text { être.aube-LOC }\end{array}$ \\
\hline АTK & wa:panokk (PT) (E) & /wâpanw-^kk/ & être.aube-LOC \\
\hline ALG & wàbanong $(\mathrm{PT})(\mathrm{E})$ & /wâbanw-^ng/ & être.aube-LOC \\
\hline OJB & $\begin{array}{l}\text { waabanong (PT) } \\
\text { ondaaban (II) (E) }\end{array}$ & $\begin{array}{l}\text { /wâbanw-^ng/ } \\
\text { /ond-âban/ }\end{array}$ & $\begin{array}{l}\text { être.aube-LOC } \\
\text { ABLATIF-être.aube }\end{array}$ \\
\hline CRB & mâchikîsikanohk (NI) (E) & /mâchi-kîsikâno-hk/ & début-journée-LOC \\
\hline CRP & $\begin{array}{l}\text { wâpanohk }(\mathrm{NI})(\mathrm{E}) \sim \\
\text { sâkâstênohk }(\mathrm{PT})(\mathrm{E}) \\
\text { âpihtâkîsikâhk }(\mathrm{PT})(\mathrm{S}) \sim \\
\text { apihtâkîsikânohk }(\mathrm{PT})(\mathrm{S}) \sim \\
\text { apihtâkîsikânotâhk }(\mathrm{PT})(\mathrm{S}) \\
\text { pissimotâhk }(\mathrm{PT})(\mathrm{S}) \\
\text { pahkisimotâhk }(\mathrm{PT})(\mathrm{O})\end{array}$ & $\begin{array}{l}\text { /wâpanw-^hk/ } \\
\text { /sâkâstêno-^hk/ } \\
\text { /âpihtâ-kîsikâ-hk/ } \\
\text { /âpihtâ-kîsikâno-^hk/ } \\
\text { /âpihtâ-kîsikâno-^tâhk/ } \\
\text { /pîsimw-^tâhk/ } \\
\text { /pahkisimw-^tâhk/ }\end{array}$ & $\begin{array}{l}\text { être.aube-LOC } \\
\text { être.la.lumière.du.jour-LOC } \\
\text { mi-journée-LOC } \\
\text { mi-journée-LOC } \\
\text { mi-journée-LOC } \\
\text { soleil-LOC } \\
\text { il.se.couche-LOC }\end{array}$ \\
\hline
\end{tabular}

Tableau 1 - Particules adverbiales et substantifs ayant le cycle du soleil comme source de lexicalisation. L'accent circonflexe indique que la voyelle précédente est allongée. 


\begin{tabular}{|c|c|c|c|}
\hline $\begin{array}{l}\text { Dialectes/ } \\
\text { langues }\end{array}$ & $\begin{array}{l}\text { Particule (PT)/Substantif } \\
\text { inanimé (NI) }\end{array}$ & $\begin{array}{l}\text { Découpe } \\
\text { morphologique }\end{array}$ & Gloses \\
\hline NSK & $\begin{array}{l}\text { chiiwaatinuuhch }(\mathrm{NI})(\mathrm{N}) \sim \\
\text { chiiwaatinuutaahch }(\mathrm{NI})\end{array}$ & $\begin{array}{l}\text { /chîwâtinw-^hch/ } \\
\text { /chîwâtinw-^tâhch/ }\end{array}$ & $\begin{array}{l}\text { vent.du.nord-LOC } \\
\text { vent.du.nord-LOC }\end{array}$ \\
\hline ILN, INN & $\begin{array}{l}\text { tshiuetin }(\mathrm{NI} / \mathrm{II})(\mathrm{N}) \\
\text { akuanutin, akualutin }(\mathrm{NI} / \mathrm{II})(\mathrm{S})\end{array}$ & $\begin{array}{l}\text { /chîwetinw/ } \\
\text { /akwâ-nûtin/ }\end{array}$ & $\begin{array}{l}\text { vent.du.nord } \\
\text { de.autre.rive-vent }\end{array}$ \\
\hline $\begin{array}{c}\text { CRN } \\
\text { (Chisasibi) }\end{array}$ & $\begin{array}{l}\text { chîwâtinûtâhch }(\mathrm{PT})(\mathrm{N} \sim \mathrm{O}) \\
\text { nikâpâhanûtâhch }(\mathrm{PT})(\mathrm{O})\end{array}$ & $\begin{array}{l}\text { /chîwâtinw-^tâhch/ } \\
\text { /nikâpâhanw-^tâhch/ }\end{array}$ & $\begin{array}{l}\text { vent.du.nord-LOC vent.de.l'ouest-LOC } \\
\text { vent.de.l'ouest-LOC }\end{array}$ \\
\hline $\begin{array}{c}\text { CRS } \\
\text { (Mistissini) }\end{array}$ & $\begin{array}{l}\text { chîwetinûtâhch }(\mathrm{PT})(\mathrm{N}) \sim \\
\text { pâshtâchîwetin }(\mathrm{NI} / \mathrm{II})(\mathrm{N}) \\
\text { chîwetinihch }(\mathrm{NI})(\mathrm{O}) \\
\text { chîwetin }(\mathrm{NI} / \mathrm{II})(\mathrm{O} \sim \mathrm{N}) \\
\text { nakâpehanûtâhch }(\mathrm{PT})(\mathrm{O})\end{array}$ & $\begin{array}{l}\text { /chîwetinw-^tâhch/ } \\
\text { /pâshtâ-chîwetin/ } \\
\text { /chîwetin-ihch/ } \\
\text { /chîwetinw/ } \\
\text { /nakâpehanw-^tâhch/ }\end{array}$ & $\begin{array}{l}\text { vent.du.nord-LOC } \\
\text { au.delà-vent.du.nord } \\
\text { vent.du.nord-LOC } \\
\text { vent.de.l'ouest-LOC } \\
\text { vent.de.l'ouest-LOC }\end{array}$ \\
\hline ATK & ki:wetinokk $(\mathrm{PT})(\mathrm{N})$ & /kîwetinw-^kk/ & vent.du.nord-LOC \\
\hline ALG & kìwedinong $(\mathrm{PT})(\mathrm{N})$ & /kîwetinw-^ng/ & vent.du.nord-LOC \\
\hline CRM & kîwetinohk $(\mathrm{NI})(\mathrm{N})$ & /kîwetinw-^hk/ & vent.du.nord-LOC \\
\hline OJB & giiwedin $($ ong $)(\mathrm{NI} / \mathrm{II})(\mathrm{N})$ & /kîwetinw/ & vent.du.nord-(LOC) \\
\hline CRB & kîwîtinohk (NI) (N) & /kîwîtinw-hk/ & vent.du.nord-LOC \\
\hline CRP & $\begin{array}{l}\text { kîwêtinohk }(\mathrm{NI}) \sim \\
\text { kîwêtinotâhk }(\mathrm{PT})(\mathrm{N}) \\
\text { nakahpêhanohk }(\mathrm{PT})(\mathrm{O})\end{array}$ & $\begin{array}{l}\text { /kîwêtinw-^hk/ } \\
\text { /kîwêtinw-^tâhk/ } \\
\text { /nakahpêhanw-^hk/ }\end{array}$ & $\begin{array}{l}\text { vent.du.nord-LOC } \\
\text { vent.du.nord-LOC } \\
\text { vent.de.l'ouest-LOC }\end{array}$ \\
\hline
\end{tabular}

Tableau 2 - Particules adverbiales de lieux et substantifs ayant le vent comme source de lexicalisation ${ }^{17}$.

\begin{tabular}{|c|c|c|c|}
\hline $\begin{array}{c}\text { Dialectes/ } \\
\text { langues }\end{array}$ & Particule & $\begin{array}{c}\text { Découpe } \\
\text { morphologique }\end{array}$ & Gloses \\
\hline \multirow[t]{2}{*}{$\begin{array}{c}\text { INN, ILN } \\
\text { (bassin hydrographique } \\
\text { du Saint-Laurent) }\end{array}$} & $\begin{array}{l}\text { mâmîht mamit } \\
\text { mâmîht utn }\end{array}$ & $\begin{array}{l}\text { /mâmîht/ } \\
\text { /mâmîht utin/ }\end{array}$ & $\begin{array}{l}\text { aval } \\
\text { de.l'aval venter } \\
\rightarrow \text { il vente de l'est }\end{array}$ \\
\hline & $\begin{array}{l}\text { natimit } \\
\text { natimit utin }\end{array}$ & $\begin{array}{l}\text { /natimit/ } \\
\text { /natimit utin/ }\end{array}$ & $\begin{array}{l}\text { amont } \\
\text { de.l'amont venter } \\
\rightarrow \text { il vente de l'ouest }\end{array}$ \\
\hline $\begin{array}{c}\text { CRN, CRS } \\
\text { (bassin hydrographique } \\
\text { de l'est de la Baie James) } \\
\end{array}$ & achishtuw & /achituw/ & aval, (à) l'ouest au nord \\
\hline $\begin{array}{c}\text { CRP } \\
\text { (bassins hydrographiques } \\
\text { de l'Alberta } \\
\text { et de la Saskatchewan) }\end{array}$ & $\begin{array}{l}\text { mâhihk } \sim \text { mâmihk } \\
\text { natamihk } \\
\text { nâtakâm }\end{array}$ & $\begin{array}{l}\text { /mâhihk/ /mâmihk/ } \\
\text { /natamihk/ } \\
\text { /nâtakâm/ }\end{array}$ & $\begin{array}{l}\text { aval, (à) l'est } \\
\text { amont, (à) l'ouest } \\
\text { autre.coté.du.rivage } \rightarrow \text { le nord }\end{array}$ \\
\hline $\begin{array}{c}\text { MSK } \\
\text { (bassin hydrographique } \\
\text { de la rivière Ohio) }\end{array}$ & $\begin{array}{l}\text { anemya } \cdot k a \\
a \cdot s a m i\end{array}$ & $\begin{array}{l}\text { /anemyâka/ } \\
\text { /âsami/ }\end{array}$ & $\begin{array}{l}\text { aval, (à) l'est } \\
\text { amont, (à) l'ouest }\end{array}$ \\
\hline
\end{tabular}

Tableau 3 - Particules adverbiales de lieux ayant le cours des rivières comme source de lexicalisation. 


\begin{tabular}{|c|c|c|c|}
\hline $\begin{array}{l}\text { Dialectes I } \\
\text { langues }\end{array}$ & $\begin{array}{c}\text { Particule (PT)/ Substantif } \\
\text { inanimé (NI) }\end{array}$ & $\begin{array}{c}\text { Découpe } \\
\text { morphologique }\end{array}$ & Gloses \\
\hline NSK & $\begin{array}{l}\text { saaun }(\mathrm{NI}) \\
\text { saaunuutaahch }(\mathrm{PT})\end{array}$ & $\begin{array}{l}\text { /sâwinw/ } \\
\text { /sâwinw-^^âhch/ }\end{array}$ & $\begin{array}{l}\text { sud } \\
\text { sud-LOC }\end{array}$ \\
\hline CRN & shâwinûtâhch (РT) & /shâwinw-^tâhch/ & sud-LOC \\
\hline CRS & $\begin{array}{l}\text { shâwanûtâhch (PT) } \\
\text { shâwan (ihch) (NI) }\end{array}$ & $\begin{array}{l}\text { /shâwanw-^tâhch/ } \\
\text { /shâwan-ihch/ }\end{array}$ & $\begin{array}{l}\text { sud-LOC } \\
\text { sud-(LOC) }\end{array}$ \\
\hline ALG & shàwanong $(\mathrm{PT})$ & /shâwanw-^ng/ & sud-LOC \\
\hline ATK & $\begin{array}{l}\text { s̀a:wanokk }(\mathrm{PT}) \\
\text { sa: } w a n w(\mathrm{NI})\end{array}$ & $\begin{array}{l}\text { /shâwanw-^hk/ } \\
\text { /shâwanw/ }\end{array}$ & $\begin{array}{l}\text { sud-LOC } \\
\text { sud }\end{array}$ \\
\hline CRM & shâwanohk (PT) & /shâwanw-^hk/ & sud-LOC \\
\hline OJB & zhaawanong (PT) & /shâwanw-^ng/ & sud-LOC \\
\hline CRB & $\begin{array}{l}\text { sâwanohk (NI) } \\
\text { sâwan (ni) }\end{array}$ & $\begin{array}{l}\text { /shâwanw-^hk/ } \\
\text { /sâwanw/ }\end{array}$ & $\begin{array}{l}\text { sud-LOC } \\
\text { sud }\end{array}$ \\
\hline CRP & sâwanohk $(\mathrm{PT})$ & /sâwanw-^hk/ & sud-LOC \\
\hline MSK & sha.wanoki (PT) & /shâwanw-^ki/ & sud-LOC \\
\hline
\end{tabular}

Tableau 4 - Particules adverbiales de lieux ayant une déitélzone comme source de lexicalisation.

\section{NOTES}

Je tiens à remercier ici mes informateurs cris pour le temps qu'ils ont consacré à m'enseigner les aspects de leur langue présentés ici. Évidemment, je suis le seul responsable pour les erreurs qui auraient pu se glisser dans l'analyse proposée. Merci à Lizeth Donoso-Herrera pour avoir aimablement traduit le résumé vers l'espagnol, de même qu'au lexicographe Kevin Brousseau pour les nombreuses discussions fructueuses que nous avons eues au cours des années au sujet des directions cardinales en cri de l'Est. Enfin, je tiens à remercier Solomon Ratt pour avoir mis à ma disposition ses données lexicographiques sur le cri des Bois.

1. Le lecteur pourra aussi consulter MacLean (1893) au sujet du mythe de création des vents chez les Blackfoot, de même que Speck (1915, p. 74) ou encore Skinner (1911, pp. 59-60) qui traite des prescriptions rituelles entourant les quatre déités éoliennes chez les Cris de l'Est. Hallowell (1955, p. 190) présente, lui aussi, une version de l'origine des quatre vents pour le saulteux (ojibwé).

2. Les abréviations linguistiques utilisées dans cet article sont les suivantes : LOC locatif ; II verbe intransitif inanimé ; NA substantif animé ; NAD nom animé dépendant ; NI substantif inanimé ; SG singulier ; PL pluriel ; PT particule adverbiale de lieu ; > donne historiquement ; 0 personne inanimée de rang troisième ; 3 personnne animée de rang troisième.

3. Afin d'éviter une discussion sur la question de la différence entre «langue » et "dialecte» - laquelle n'aurait d'ailleurs pas sa place dans une étude comme celle-ci-, nous utilisons le terme « dialecte » dans un sens relâché. Par exemple, nous considérons que le cri de l'Est (dialecte du Nord) est composé de deux sous-dialectes. Celui utilisé à Chisasibi, Wemindji et Eastmain, et un autre sous-dialecte utilisé à Whapmagoostui. L'ojibwé et l'algonquin sont des langues différentes du cri.

4. Dans les langues algonquiennes, l'obviation est une sous-catégorie de la catégorie grammaticale de la personne qui permet de faire une distinction entre deux personnes troisièmes (animées ou non). En outre, l'obviation permet de garder une trace de la configuration entre troisièmes personnes et relations grammaticales (sujet, objet) au sein du mot, de la phrase ou du discours. L'obviation opère selon un principe simple et très souple : quand deux personnes animées de rang ordinal troisième sont 
mises en relation - au sein d'un discours, d'une ou de plusieurs phrases, d'un groupe ou d'un mot -, l'obviation est déclenchée par la deuxième personne animée. Une seule de ces personnes est la personne dite « rapprochée », c'est-à-dire qu'elle occupe le foyer ou le premier plan du discours. La ou les autres personnes de rang troisième occupent automatiquement le deuxième (personne « obviée ») ou le troisième plan du discours (personne «surobviée »).

5. Les particules ou adverbes modifient un verbe et ne se fléchissent pas (Hockett 1939, p. 241 ; Drapeau 2014, chap. 20). Elles sont dérivées ou non, et, dans ce dernier cas, elles sont indécomposables. Leur position dans la phrase est relativement libre puisqu'elles peuvent survenir aussi bien en dehors du complexe verbal qu'à l'intérieur de celui-ci.

6. Le Père Bonaventure Fabvre note, dans ses Racines Montagnaises (manuscrit publié avant 1695), les mots 8aban « Le point I8r, Aurore », 8abanatak « estoille du point du I8r, Lucifer », ainsi que le préfixe 8aban - qui signifie qu'on passe la nuit à faire quelque chose (1970 [1695], pp. 208-209). Le chiffre 8 indique une voyelle ronde antérieure et fermée $/ u /$.

7. Il est possible que ce mot désignait à l'origine Vénus (l'étoile du soir), comme semble l'indiquer Johnston (1976, p. 31), mais aussi, par métonymie, la direction OUEST, puisque Couture (1982, p. 37) rapporte pour l'algonquin de l'Abitibi la particule nêgabianouk «à l'est» - et on sait que Vénus apparaît autant à l'est (matin) qu'à l'ouest (soir). Il est probable que cette utilisation soit une innovation secondaire puisqu'elle est confinée à ce dialecte. En synchronie, l'ojibwé présente le substantif animé onaagoshi'anang « Vénus, l'étoile du soir » (NA) (dont la composition interne est onaagoshi- " soir » $+/ \mathrm{y}$ ou $\%$-anang « étoile »).

8. Nous remercions la linguiste Megan Lukaniec de nous avoir mis au fait de ce détail.

9. Pour le mesquakie, Jones $\left(1939\right.$, p. 21) note que $C \bar{a} w a n \bar{o}^{a}$ est le vis-à-vis méridional de $W \bar{i} s a$ 'kä qui habite au nord.

10. L'introduction du mythe ojibwé de la création des quatre vents (ou déités cardinales/éoliennes) est tout à fait claire à ce sujet; il s'agit d'une mère qui veut protéger sa fille fragile du mal et lui interdit de faire face à l'ouest : "[...] she was told in a dream that the child should never at any time face the place of the going down of the sun. " (Radin et Reagan 1925, p. 106). Le tabou, et l'évitement culturel qui le motive, est un facteur de changement sémantique en linguistique historique, et les expressions frappées d'un tabou - comme dans le cas qui nous concerne ouEST $=$ monde des morts $=$ brunante - ont tendance à être évitées au moyen de stratégies diverses (métaphore, euphémisme ou circonlocution descriptive). Il peut même arriver que ces stratégies deviennent elles-mêmes interdites ; il s'en suit alors une chaîne de remplacements qui provoque d'importantes modifications dans un champ lexical donné (Hock 1991, p. 293). Ces règles culturelles ont peut-être eu un effet sur le chevauchement et le remplacement des termes pour OUEST dans les dialectes du continuum CINA.

11. Une de mes informatrices du cri de l'Est (dialecte du Sud), originaire de Nichikun (Qc.), affirmait que le terme chishechîwetin « il vente du nord » (avec le préfixe chishe- " grand, majestueux, vieux ») avait une connotation plus animiste que chîwetin. Elle se rappelait que son père l'utilisait régulièrement avant de devenir pasteur pour sa communauté.

12. On retrouve un mot similaire chez Fabvre (1970 [1695], p. 41), atimipâri8 « Le Iour s'abaisse, decline ».

13. Selon Rogers (1963, p. 8), les vents provenant du nord-ouest, de l'ouest et du sud-ouest dans la région du lac Mistissini sont prédominants tout au long de l'année. En hiver, le vent du nord-ouest s'accompagne de températures très froides, d'un ciel dégagé et d'un temps sec.

14. Nous avons noté qu'en contexte cérémoniel, chîwetin « il vente du nord/le Nord » est souvent en rapport de relative synonymie avec les substantifs animés nimushum « mon grand-père » (NAD) et kâkûsh «ours noir» (NA). Ces trois mots qui invoquent l'idée de sagesse sont répétés ensemble par le célébrant.

15. Ce symbolisme anthropomorphique entre NORD-AîNÉ (Wīsa'kä) et OUEST-CADET (Iyāpā'tä) se retrouve aussi dans des portions de l'imposante mythologie mesquakie (algonquien du Centre). Jones (1939, p. 16) rapporte ce symbolisme comme suit : "Iyāpā'tä is younger brother to Wīsa'kä and he dwells in a lodge in the spirit world. This place is at the west and beyond where the sun goes down. [...] 
Mortals go beyond the setting sun when they die. They stay about the earthly home 4 days, and then go west along a deep, narrow path until they come to a river which flows along with great rapidity. "

16. Les données personnelles de l'auteur ont été recueillies lors de plusieurs séjours à Chisasibi et à Whapmagoostui entre 2009 et 2011, au moyen de conversations et d'interrogations directes avec quatre locuteurs masculins âgés entre 21 et 71 ans. Plusieurs données proviennent toutefois d'observations en contexte cynégétique. Les données concernant le cri de l'Est (dialecte du Nord) parlé à Whapmagoostui ont été revérifiées avec deux locuteurs âgés et diffèrent quelque peu de celles de Chisasibi.

17. La palatalisation de $* / \mathrm{k} / \mathrm{en} / \mathrm{t} \mathrm{f} /$ (ce dernier phonème étant orthographié $c h$ ou $t s h$ dans le présent article) permet de tracer une ligne isoglossique entre les dialectes cris de l'Ouest canadien et ceux du Québec (mis à part l'atikamekw), au sein du continuum CINA.

\section{RÉFÉRENCES CITÉES}

BÉLAND Jean-Pierre

1978 Atikamekw morphology and lexicon, thèse de doctorat, University of California, 2 vol., $651 \mathrm{p}$.

BLOOMFIELD Leonard

1946 "Algonquian ", in H. Hoijer, C. Osgood (éd.), Linguistic structures of Native America, Viking Fund Publication in Anthropology, New York, vol. 6 , pp. 85-129.

1962 The Menomini language, Yale University Press, New Haven/Londres.

1970 Le Langage, Payot, Paris [1933].

Bobbish-Salt Luci, Alice Duff, Marie-Odile Junker, Marguerite Mackenzie (éd.)

2004 Eastern James Bay Cree dictionary (Northern dialect), Cree School Board, Cree Programs, Chisasibi, Québec.

BRAUNER Siegmund

1998 "Directions/spatial orientations in African languages: further cases", in P. Zima (éd.), Space and direction in languages, Institute of advanced studies at Charles University and the Academy of Sciences of the Czech Republic, Prague, pp. 66-78.

BRINTON Daniel G.

1868 The myths of the New World: a treatise on the symbolism and mythology of the Red Race of America, Leypoldt \& Holt, New York.

Brousseau Kevin, Vincent Collette

2014 A Dictionary of Moose Cree, Cree/English, Moose Cree First Nation/Cree Nation Government, Oujé-Bougoumou, Québec/Moose Factory, Ontario.

BRown Cecil H.

1983 «Where do cardinal direction terms come from? », Anthropological linguistics, 25, pp. 121-161.

Brown Jennifer H. S.

1977 « James Settee and his cree tradition: an Indian camp at the mouth of the Nelson River Hudsons Bay », in W. Cowan (éd.), Actes du huitième congrès des Algonquinistes, Carleton University Press, Ottawa, pp. 36-49. 
Collette Vincent

2014 Description de la morphologie grammaticale du cri de l'Est (dialecte du Nord, Whapmagoostui), thèse de doctorat, université Laval (Québec).

Coulter Charles Russel, Patricia Turner

2000 Encyclopedia of Ancient Deities, McForland \& Company Inc. Publishers, Jefferson (N.C.)/Londres.

Couture Yvon $\mathrm{H}$.

1982 Lexique français-algonquin, Éditions Hyperborées, Val d'Or.

DAY Gordon M.

1994 Western Abenaki Dictionary, Canadian ethnology service, Canadian Museum of Civilization, Hull.

DinNISON Bonnie (éd.)

1999 Atikamekw nehirowi itewin masinahikan, Conseil de la nation atikamekw, La Tuque.

DraPEAU Lynn

1991 Dictionnairemontagnais-français, Presses del'université du Québec, Québec. 2014 Grammaire de la langue innue, Presses de l'université du Québec, Québec.

DraPeAu Lynn, Renée LAMBERT-BRÉTIÈRE

2011 «Verbal classifiers in Innu », Anthropological linguistics, 53 (4), pp. 293-322.

Evans Nicholas, Hans-Jürgen SASSE

2002 «Introduction: problems of polysynthesis », in N. Evans, H.-J. Sasse (éd.), Problems of polysynthesis, Akademie Verlag, Berlin, pp. 1-13.

FABVRE Bonaventure

1970 Racines montagnaises, Centre d'études nordiques, Université Laval, Québec [1695].

FeIT Harvey

1978 Waswanipi realities and adaptations: resource management among Subarctic hunters, thèse de doctorat, université McGill (Montréal).

GODDARD Ives

1983 " The eastern algonquian subordinate mode and the importance of morphology », International Journal of American linguistics, 49 (4), pp. 351-387.

1990 "Primary and secondary stem derivation in Algonquian », International Journal of American linguistics, 56 (4), pp. 449-483.

Gutmans Théodore

1970 «Une terminologie occidentale unifiée dès le Moyen Âge : les quatre points cardinaux ", La Linguistique, 6 (1), pp. 147-151.

HaLe Kenneth

1989 « On configurational structures », in M. László, P. Myusken (éd.), Configurationality: the typology of asymmetries, Foris, Dordrecht, pp. 292-300.

Hallowell A. Irving

$1955 \quad$ Culture and experience, University of Pennsylvania Press, Philadelphie. 
HeINE Bernd

1997 Cognitive foundations of grammar, Oxford University Press, New York/ Oxford.

HEWSON John

1993 A computer-generated dictionary of proto-algonquian, Canadian Museum of Civilization, Ottawa.

Hock Hans Henrich

1991 Principles of historical linguistics, Mouton de Gruyter, Berlin/New York/ Amsterdam.

Hockett Charles F.

1939 «Potowatomi Syntax », Language, 15, pp. 235-248.

Johnston Basil

1976 Ojibway Heritage, McClelland and Stewart, Toronto.

JONES William

1911 «Notes on the Fox Indians », The Journal of American folklore, 24 (92), pp. 209-237.

1916 "Ojibwa tales from the North Shore of Lake Superior », The Journal of American folklore, 29 (113), pp. 368-391.

1939 Ethnography of the Fox Indians, M. Welpley Fisher (éd.), Smithsonian Institution, Bureau of American Ethnology, Washington (D.C.).

LACOMBE Albert

1874 Dictionnaire (grammaire) de la langue des Cris, C. H. Beauchemin et Valois, Montréal.

Lemoine Geo, O.M.I.

1909 Dictionnaire français-algonquin, G. Delisle, Chicoutimi.

LEVINSON C. Stephen

1996 «Frames of reference and Molyneux's questions: crosslinguistic evidence », in P. Bloom, M. F. Garrett, L. Nadel, M. A. Peterson (éd.), Language and space, The MIT Press, Cambridge, pp. 110-169.

MacKenZIE Marguerite

1980 Towards a dialectology of Cree-Montagnais-Naskapi, thèse de doctorat, université de Toronto.

MacKenZIE Marguerite, Bill JanCEWICZ

1994 Naskapi lexicon $=$ Lexique naskapi, Naskapi Development Corp. /Société de développement des Naskapis, Kawawachikamach, Québec.

MaCLEAN John

1893 «Blackfoot mythology», The Journal of American folklore, 6 (22), pp. 165-172.

MCGREGOR Ernest

1994 Algonquin lexicon, Kitigan Zibi Education Council, Maniwaki. 
McNulty Gérard E., Marie-Jeanne Basile

1981 Lexique montagnais-français du parler de Mingan, Centre d'études nordiques, Université Laval, Québec.

Mietzner Angelika, Helma Pasch

2007 «Expressions of Cardinal directions in Nilotic and in Ubangian languages », Journal of theoretical linguistics, 4 (3), pp. 1-16.

NeACAPPo Mimie

2012 "Land Talk» in Iiyiyiuyimuwin (Eastern James Bay Cree), mémoire de maîtrise, université Carleton (Ottawa).

Neeposh Ella, Daisy Moar, Ruth SALt, Marguerite MacKenzie, Bill Jancewicz

2004 Eastern James by cree dictionary (Southern Dialect), Cree School Board, Cree Programs, Chisasibi (Québec).

Nichols John D., Earl NyHolm

1995 A concise dictionary of Minnesota Ojibwe, Minnesota University Press, Minneapolis.

Pearson L. Bruce

1987 «Savannah and Shawnee: the end of a mini-controversy », International Journal of American linguistics, 53 (2), pp. 183-193.

Potier Pierre

1920 [1751] «Radices Huronicae», in D. Fraser (éd.), Huron manuscripts from Rev. Pierre Potier's Collection, Ontario Bureau of Archives Report, Ottawa, vol. 15, pp. 159-455.

Proulx Paul

1980 «The linguistic evidence on Algonquian prehistory », Anthropological linguistics, 22 (1), pp. 1-21.

1984 «Proto-Algonquian *aye and its implications », International Journal of American linguistics, 50 (1), pp. 84-93.

1986 "Algonquian cardinal directions », Algonquian and Iroquoian linguistics, $11(2)$, pp. 19-23.

Radin Paul, Albert B. REAGAN

1928 "Ojibwa myths and tale. The Manabozho Cycle », The Journal of American folklore, 41 (159), pp. 61-146.

Rogers Edward S.

1963 The hunting group-hunting territory complex among the Mistassini Indians, National Museum of Canada (Bulletin, 195), Ottawa.

SAPIR Edward

1970 Le Langage. Introduction à l'étude de la parole, Petite Bibliothèque Payot, Paris [1921].

SIEBERT Frank T.

1967 The original home of the Proto-Algonquian people, National Museum of Canada (Bulletin, 214), Ottawa. 
SiLvy Antoine

1974 Dictionnaire montagnais-français, transcription A. Lorenzo, D. E. Cooter, G. McNulty, Presses de l'université du Québec, Montréal [1678].

SKINNER Alanson

1911 Notes on the Eastern Cree and Northern Saulteaux, Order of the Trustees, vol. IX, part I, published by order of the Trustees [of the American Museum of Natural History], New York.

SPECK Frank G.

1915 «Some Naskapi Myths from Little Whale River », The Journal of American folklore, 28 (107), pp. 70-77.

SWADESH Morris

2006 The origin and diversification of languages, Aldine Transactions, New Brunswick/Londres [1971].

TANNER Adrian

1979 Bringing home animals. Religious ideology and mode of production of the Mistassini Cree hunters, Londres/St. John's/New York, Hurst Publishers/ Institute of social and economic research/St. Martin's Press.

VALENTINE Randolph

2001 Nishnaabemwin reference grammar, University of Toronto Press, Toronto.

Wolfart Christopher, Freda AhEnaKew

1998 The student's dictionary of literary Plains Cree, based on contemporary texts, Algonquian and Iroquoian Linguistics, Winnipeg.

WoLVENGREY Arok

2001 Nêhiyawêwin: itwêwina, Cree Words, Canadian Plains research center, University of Saskatchewan, Regina (Saskatchewan).

\section{SOURCES ÉLECTRONIQUES}

2003-2014 Cheyenne, Cheyenne Dictionary, http://cdkc.edu/cheyennedictionary/index.html 2014 Cri de l'Est, http://www.atlas-ling.ca/

2014 Ojibwé, The Ojibwe People's Dictionary, http://ojibwe.lib.umn.edu/

2013 Innu, http://www.innu-aimun.ca/english/dictionary/ 